\title{
Analysis of the internal electric fields of pristine ice crystals and aggregate snowflakes, and their effect on scattering
}

Article

Accepted Version

Creative Commons: Attribution-Noncommercial-No Derivative Works 4.0

McCusker, K., Westbrook, C. D. and Moiola, A. (2019) Analysis of the internal electric fields of pristine ice crystals and aggregate snowflakes, and their effect on scattering. Journal of Quantitative Spectroscopy and Radiative Transfer, 230. pp. 155-171. ISSN 0022-4073 doi:

https://doi.org/10.1016/j.jpsrt.2019.04.019 Available at https://centaur.reading.ac.uk/83301/

It is advisable to refer to the publisher's version if you intend to cite from the work. See Guidance on citing.

To link to this article DOI: http://dx.doi.org/10.1016/j.jqsrt.2019.04.019

Publisher: Elsevier

All outputs in CentAUR are protected by Intellectual Property Rights law, including copyright law. Copyright and IPR is retained by the creators or other copyright holders. Terms and conditions for use of this material are defined in the End User Agreement. 


\section{CentAUR}

Central Archive at the University of Reading

Reading's research outputs online 


\title{
Analysis of the internal electric fields of pristine ice crystals and aggregate snowflakes, and their effect on scattering.
}

\author{
K. McCusker ${ }^{\mathrm{a}, *}$, C.D. Westbrook ${ }^{\mathrm{a}}$, A. Moiola ${ }^{\mathrm{b}}$ \\ ${ }^{a}$ Department of Meteorology, University of Reading \\ ${ }^{b}$ Department of Mathematics, University of Pavia
}

\begin{abstract}
The discrete dipole approximation is used to explore the internal electric fields of plane-wave-illuminated ice particles, and analyse their differential scattering cross sections. The results are displayed for monocrystals and aggregates of size parameters $x=2$ and $x=10$. We show that the field is relatively uniform for $x=2$, but for monocrystals of $x=10$ there is a complex internal structure. For a hexagonal plate, this structure is a combination of two components: a "distorted" plane wave, with wavefronts aligned perpendicular to the incident wave close to the centre of the plate, and curved forward near the particle boundary; and a standing wave, internally reflected around the perimeter. The former is due to the transverse component of the field i.e., the component perpendicular to the incident wave, and the latter is due to the component parallel to the incident direction. Focussing of the field towards the forward side of the particle is observed. As the particle complexity is increased due to aggregation, the field becomes smoother and less focussing is seen. For complex aggregates, the individual monomers act independently of one another, suggesting simplified methods of calculating scattering from such particles. The influence of the internal fields on far-field scattering is explored. It is demonstrated that scattering in the forward and backward directions is dominated by the transverse component. The parallel component contributes to sidescattering, with its influence on total scattering decreasing with particle complexity. We propose that this is due to the inability of complex particles to maintain a standing wave, diminishing much of the sidescattering observed for monocrystals. Comparisons of the far-field scattering properties of complex aggregates using the discrete dipole and Rayleigh-Gans approximations are also presented for $x=2$ and $x=10$, along with results obtained using a soft sphere approximation.
\end{abstract}

Keywords: discrete dipole approximation, electromagnetic scattering, ice, snowflakes, microwave, sub-millimeter

\section{Introduction}

Ice particles in clouds have important radiative and hydrological impacts, contributing to rainfall in the midlatitudes [1], and snowfall at high latitudes. To explore these processes in more detail, measurements of microphysics are required. This is made possible using scattered electromagnetic waves retrieved by remote sensing instruments such as radars and radiometers.

Numerical Weather Prediction (NWP) and climate models have benefitted greatly from developments in remote sensing instruments and the wide variety of measurements they permit. However, there is still work to be done to improve ice cloud retrievals in order to gain more precise information on cloud and hydrometeor profiles. For example, comparing 5 different satellite products, Refs. [2,3] have shown that there is an enormous spread between retrieved ice water path from the different datasets. Improved retrievals of such properties are integral to the development of ice cloud microphysics schemes, which will allow advancements in the representation of different weather

\footnotetext{
${ }^{*}$ Corresponding author

Email address: karina.mccusker@pgr.reading.ac .uk (K. McCusker )
}

and climate processes in models. Furthermore, they will enable more accurate detection of snowfall from space, which remains an area of great difficulty. CloudSat [4] has provided our best estimates thus far of global snowfall, using a $94 \mathrm{GHz}$ cloud radar to profile cloud vertical structure and obtain information on the constituent ice and water particles. However, there are still substantial uncertainties in converting the backscatter from snow at this frequency into a snowfall rate, one of which is the accuracy of the scattering [5].

Efforts have been made to improve airborne and spaceborne retrievals of ice water path by measuring sub-mm brightness temperatures using radiometers such as CoSSIR, ISMAR, and ICI $[6,7,8]$. ICI has been specifically designed for observing cloud ice, and will be the first operational instrument to cover sub-millimeter wavelengths, with frequencies ranging from 183 to $664 \mathrm{GHz}$. However, retrievals from these instruments are sensitive to scattering by ice particles which are comparable to or larger than the wavelength.

In the past, radiative transfer models have employed drastic simplifications of cloud ice, such as approximating particles by spheres or spheroids of equivalent size. However, as particle size increases with respect to the wavelength, the particle shape and material play a significant role in different interference pat- 
terns that are found within the particle. Recently there have been advances in using more realistic habits for single scattering calculations e.g. the ARTS database [9, 10]. With the advent of high frequency observations, accurate retrievals require continued efforts to improving our understanding of the scattering properties of realistic ice cloud particles. This will enable better interpretations of scattered electromagnetic waves, in turn allowing more precise retrievals of cloud properties from both radars and radiometers.

The single-scattering properties of various particle geometries are typically explored using numerical methods such as the discrete dipole approximation (DDA), and the RayleighGans approximation (RGA). The starting point of both methods is to discretise the particle into small volume elements, each of which is excited by an electric field. Accurate calculations are possible using DDA, but this technique is computationally expensive as it includes interactions between different volume elements. Less expensive methods such as RGA have been employed by researchers in an attempt to calculate scattering properties in a more efficient manner, such as in Ref. [11]. The field at each element in RGA is approximated by the applied field, with no interactions experienced between volume elements. Hence RGA is applicable for cases where interactions are negligible, i.e. when the relative refractive index is close to unity, and the phase shift across the particle is small. However, comparing to DDA results of the far-field scattering properties of snowflakes, Tyynelä et al. [12] found that RGA can lead to relative biases of $-65 \%$. Leinonen et al. [13] also found that even larger biases can occur for ice particles with elevated density due to riming. These findings suggest that the electric field inside the snowflake may be systematically larger in magnitude than the incident wave.

The purpose of the present study is to explore the scattering of $\mathrm{mm}$ and sub-mm electromagnetic waves by ice particles through an investigation of their internal electric fields, rather than their far-field scattering properties. As we will show, this allows us to identify different physical effects, which in turn control different aspects of the far-field scattering; in addition it allows us to test at a more fundamental level the realism of approaches like RGA, by comparing the true internal field with the incident plane wave.

Internal fields of spheres, spheroids and cylinders have been explored in other disciplines, such as nanophotonics [14, 15]. In these studies, complex internal field structures are seen, with constructive interference within the particles leading to a region of high electric field magnitude at the shadow-side of the particle, similar to the characteristic focussing nature of a lens. Owen et al. [16] describe the internal fields in infinitely long dielectric cylinders for size parameters of order 40. They observed enhanced electric fields in the forward portion of the cylinder which they ascribe to geometrical focussing; in addition, they noted the existence of a partial standing wave pattern close to the boundary of the scatterer. These surface waves are present over a range of size parameters: they are greatly enhanced in magnitude at very specific size parameters corresponding to resonances, or whispering gallery modes, where waves are internally reflected around the perimeter of the cylinder and repeat themselves (matched phase) after each trip; but they are also present as "partial standing waves" in the offresonant case. Ref. [17] also modelled similar extrema in wavelength-scale spherical particles.

Some research has been done on the internal field of more irregular particles $[18,19]$. Similar focussing behaviour of the field was observed for Gaussian random spheres and debris particles, with the amount of focussing decreasing with increased shape complexity [18]. It was shown by Barton [19] that internal field variations with particle geometry lead to significant differences in far-field scattering properties. Lu et al. [20] studied the field inside a dendritic ice crystal, using their findings to modify RGA in such a way that scattering calculations are improved by including short-range interactions between volume elements. However, few other researchers have studied the problem for realistic ice particles to explore how these complex internal field structures differ with shape, and the role these variations play in far-field scattering. We hope to address the problem at a fundamental level to acquire understanding of how scattering at radar and radiometer frequencies works.

\section{Atmospheric ice particles}

The constituent particles of ice clouds vary in size and shape depending on the temperature and supersaturation of the surrounding environment. Different growth processes complicate the crystal habits, such as diffusion, accretion, and aggregation. Aggregation can occur due to collision and coalescence, resulting in complex snowflake shapes.

We will investigate a number of different idealisations of single and aggregated ice crystals. These are:

(a) Hexagonal plates: Horizontally aligned hexagonal plates are frequently found in mixed-phase layer clouds [21], and at the top of cirrus clouds [22, 23], particularly in anvil cirrus [24]. These studies found plate-like crystals with maximum dimensions reaching almost $1 \mathrm{~mm}$.

(b) Hexagonal columns: Collating data from three different cloud campaigns, Um et al. [24] concluded that hexagonal columns exist at all temperatures explored, between -87 and $0^{\circ} \mathrm{C}$. However, growth is favoured between -3 and $-8^{\circ} \mathrm{C}$. During the campaigns, columns of projected maximum dimensions between approximately $0.025 \mathrm{~mm}$ and $1 \mathrm{~mm}$ were observed. In this study we look at short columns of solid ice, which usually occur at low ice supersaturations [25].

(c) Chain aggregates of plates: Aggregates of plate-like crystals occur in regions of high electric field, such as within deep convective clouds [26, 27, 28]. This particle shape is a logical elaboration on single plates and is useful to study the transition from single crystals to complex aggregates.

(d) Irregular aggregates of ice crystals: These are a more complex representation of realistic aggregates found in stratiform ice clouds and snowfall. Hobbs et al. [29] observed dendritic aggregates of maximum dimensions up 
to $1.4 \mathrm{~cm}$. Such large aggregates play a significant role in microwave scattering as they dominate radar reflectivity when present, thus proving important for snowfall retrievals. Hence it is useful to explore their scattering properties. In this study we present results for two aggregates of fernlike dendrites modelled by Tyynelä et al. [30].

\section{Modelling particles}

We define the size parameter of a particle as $x=k D_{\max } / 2$, where $k=\frac{2 \pi}{\lambda}$ is the wavenumber corresponding to an incident wave of wavelength $\lambda$. $D_{\text {max }}$ is the maximum distance between any 2 points within the particle. The frequency range covered by ISMAR and ICI is $83-874 \mathrm{GHz}$. We are also interested in radar frequencies of $3-94 \mathrm{GHz}$. Therefore, the range of wavelengths relevant to this study is $0.34 \mathrm{~mm}-10 \mathrm{~cm}$.

We assume that ice is an insulator with magnetic permeability $\mu=1$. This means the electric properties of the material are determined completely by the complex relative permittivity $\epsilon=\epsilon_{r}+i \epsilon_{i}$, which we assume is constant throughout the medium. We can also write $\epsilon=m^{2}$, where $m$ is the complex index of refraction of the particle. As recommended by Eriksson et al. [31], the permittivity parameterisation introduced by Mätzler [32] has been used to calculate the dielectric properties of the particles modelled in this study. The real part of the permittivity $\epsilon_{r}$ (dielectric constant), which represents phase velocity, varies weakly with temperature. The imaginary part $\epsilon_{i}$, which represents absorption of the wave in the particle, varies more strongly with temperature, and also with frequency. In the calculations presented here, we have assumed a temperature of $-20^{\circ} \mathrm{C}$.

For the irregular aggregates of crystals studied in this paper, comparisons have been done using the Maxwell-Garnett effective medium approximation [33]. Using this method, irregular particles are approximated by simpler shapes comprised of a homogeneous ice-air mixture; in this study we have used spheres of equal $D_{\max }$ to the aggregate. The effective permittivity is determined based on the volume fraction of ice within the sphere.

We discretise an integral formulation of Maxwell's equations (DDA) and numerically solve the discrete system. This is done at two different frequencies for each particle to calculate the internal electric field for size parameters of $x=2$ and $x=10$. For these properties the real part of $\epsilon$ has a value of 3.1702 for solid ice, and the imaginary part varies between 0.0002 and 0.0075 . We also present calculations for some particles with a size parameter of $x=0.01$. The corresponding wavelength of these particles result in an imaginary part of $\epsilon$ which is of order $10^{-6}$.

The aspect ratio of the monocrystals is defined as the ratio between the length of the particle in the $z$ direction and the maximum width of the particle in the $x-y$ plane. The monocrystals presented in this manuscript have a fixed orientation. The incident plane wave is polarised in the $x$ direction and travels in the direction of the $y$-axis. Some additional scenarios using a $z$ polarised plane wave and other particle orientations have been explored, with these results presented in the supplementary material for interested readers. Orientationally averaged far-field scattering quantities are considered for aggregates in section 7.

\section{Discrete Dipole Approximation}

Many different numerical methods have been applied to solve electromagnetic scattering problems. The discrete dipole approximation is a flexible method that is commonly used to approximate the scattering properties of penetrable objects of arbitrary geometry $[34,35]$. In this method, a particle is approximated by $N$ polarisable volume elements (or dipoles) located in a cubical array. Each dipole $j(=1, \ldots, N)$ has a polarisation $\mathbf{P}_{j}=\alpha_{j} \mathbf{E}_{j}^{\text {exc }}$, where $\alpha_{j}$ is the polarisability. There are different ways to prescribe the polarisability $\alpha_{j}$ of the dipoles that represent the particle. In this study we use the Lattice Dispersion Relation (LDR), derived by Ref. [36]. We illuminate the particle with a plane wave:

$$
\mathbf{E}_{j}^{i n c}=\mathbf{E}_{0} \exp \left(i \mathbf{k} \cdot \mathbf{r}_{j}-i \omega t\right) .
$$

$\mathbf{E}_{0}=E_{0} \hat{\mathbf{e}}$ is the polarisation vector for the electric field, where $\hat{\mathbf{e}}$ is the unit vector in the direction of polarisation. We assign a unit amplitude, $E_{0}=1$. $\mathbf{k}$ is the wave vector, whose magnitude $k=|\mathbf{k}|$ is the wavenumber, and whose direction $\mathbf{k} / k$ is the direction of propagation of the incoming wave. $\mathbf{r}_{j}$ is the position of dipole $j$, and $\omega$ is the angular frequency. From here on in, we assume all fields are time-harmonic, thus leaving out the $\exp (-i \omega t)$ component. The electric field incident on each dipole (the "exciting" field) $\mathbf{E}_{j}^{e x c}$ is given by $\mathbf{E}_{j}^{i n c}$, plus the contributions from each of the other dipoles in the particle:

$$
\mathbf{E}_{j}^{e x c}=\mathbf{E}_{j}^{i n c}-\sum_{j^{\prime} \neq j} \mathbf{A}_{j j^{\prime}} \mathbf{P}_{j^{\prime}} .
$$

$\mathbf{A}$ is a $3 N \times 3 N$ array commonly referred to as the interaction matrix. Each entry $\mathbf{A}_{j j^{\prime}}$ is a $3 \times 3$ matrix which can be calculated for $j \neq j^{\prime}$ by:

$$
\mathbf{A}_{j j^{\prime}}=\frac{\exp (i k R)}{R}\left[k^{2}\left(\hat{\mathbf{R}} \hat{\mathbf{R}}-\mathbf{1}_{3}\right)+\frac{i k R-1}{R^{2}}\left(3 \hat{\mathbf{R}} \hat{\mathbf{R}}-\mathbf{1}_{3}\right)\right]
$$

for $j, j^{\prime}=1, \ldots, N$. The distance between points $\mathbf{r}_{j}$ and $\mathbf{r}_{j^{\prime}}$ is given by $R=|\mathbf{R}|$, where $\mathbf{R}=\mathbf{r}_{j}-\mathbf{r}_{j^{\prime}}$. $\hat{\mathbf{R}}=\mathbf{R} / R$ is the directional unit vector between the points, and $\hat{\mathbf{R}} \hat{\mathbf{R}}$ is a dyadic formed from these interdipole unit vectors. Recall $\hat{\mathbf{R}} \hat{\mathbf{R}}$ is a $3 \times 3$ complex matrix whose element $(\hat{\mathbf{R}} \hat{\mathbf{R}})_{J, J^{\prime}}=\hat{\mathbf{R}}_{J} \hat{\mathbf{R}}_{J^{\prime}}$ for $J, J^{\prime}=$ $1,2,3$. As in Ref. [35], we make the standard assumption that $\mathbf{A}_{j j}=\alpha_{j}^{-1} \mathbf{1}_{3}$, where $\mathbf{1}_{3}$ is the $3 \times 3$ identity matrix. Thus we can write $\mathbf{E}_{j}^{e x c}=\mathbf{A}_{j j} \mathbf{P}_{j}$ and rearrange (2) as:

$$
\mathbf{E}_{j}^{i n c}=\mathbf{A}_{j j} \mathbf{P}_{j}+\sum_{j^{\prime} \neq j} \mathbf{A}_{j j^{\prime}} \mathbf{P}_{j^{\prime}} .
$$

Hence the scattering problem can be reduced to a system of $3 \mathrm{~N}$ linear equations to solve for the unknown dipole polarisations $\mathbf{P}_{j^{\prime}}$ : 


$$
\sum_{j^{\prime}=1}^{N} \mathbf{A}_{j j^{\prime}} \mathbf{P}_{j^{\prime}}=\mathbf{E}_{j}^{i n c} .
$$

Once the value of $\mathbf{P}_{j}$ is known for each dipole, it is straightforward to compute the macroscopic electric field inside the volume elements:

$$
\mathbf{E}_{j}=\frac{\mathbf{P}_{j}}{V_{j} \chi_{j}}
$$

where $V_{j}=d^{3}$ is the volume of the dipole and $\chi_{j}=(\epsilon-1) / 4 \pi$ is the susceptibility of the medium [37]. This should not be confused with the exciting electric field $\mathbf{E}_{j}^{\text {exc }}=\mathbf{P}_{j} / \alpha_{j}$ mentioned previously, which includes the field resulting from the incident wave and contributions from the other $N-1$ dipoles, but not the field induced by the dipole on itself. For the particles in this study, the ratio $\alpha_{j} / V_{j} \chi_{j}$ between $\mathbf{E}_{j}$ and $\mathbf{E}_{j}^{\text {exc }}$ is approximately 0.58 .

It is also possible to calculate different scattering properties of the particle, such as the scattered electric field. This is done by summing the power radiated by the array of $N$ oscillating dipoles. If the distance $r$ between a detector and scatterer is large, the scattered far field can be approximated by [35]:

$$
\mathbf{E}_{s c a}(\mathbf{r})=\frac{\exp (i k r)}{r} k^{2}\left(\hat{\mathbf{n}} \hat{\mathbf{n}}-\mathbf{1}_{3}\right) \sum_{j=1}^{N} \mathbf{P}_{j} \exp \left(-i k \mathbf{r}_{j} \cdot \hat{\mathbf{n}}\right),
$$

where $\hat{\mathbf{n}}=\mathbf{r} / r$ is the unit vector in the scattering direction, and $\hat{\mathbf{n}} \mathbf{n}$ is a dyadic. From the detector, we can measure waves polarised parallel to the unit vector $\hat{\mathbf{e}}_{d e t}$, so the field we sample is $\mathbf{E}_{d e t}\left(\mathbf{r}, \hat{\mathbf{e}}_{d e t}\right)=\mathbf{E}_{s c a}(\mathbf{r}) \cdot \hat{\mathbf{e}}_{d e t}$. The normalised differential scattering cross section $\sigma\left(\hat{\mathbf{n}}, \hat{\mathbf{e}}_{d e t}\right)$ for one direction and polarisation can be calculated such that it is independent of distance $r$. The vector $\hat{\mathbf{e}}_{d e t}$ is chosen to be perpendicular to $\hat{\mathbf{n}}$, so $\hat{\mathbf{n}} \cdot \hat{\mathbf{e}}_{d e t}=0$, and we have:

$$
\sigma\left(\hat{\mathbf{n}}, \hat{\mathbf{e}}_{d e t}\right)=r^{2}\left|\mathbf{E}_{d e t}\left(\mathbf{r}, \hat{\mathbf{e}}_{d e t}\right)\right|^{2}=k^{4}\left|\sum_{j=1}^{N} \mathbf{P}_{j} \cdot \hat{\mathbf{e}}_{d e t} \exp \left(-i k \mathbf{r}_{j} \cdot \hat{\mathbf{n}}\right)\right|^{2} .
$$

If $\hat{\mathbf{n}}=-\mathbf{k} /|\mathbf{k}|$, then we obtain backscattering and $\sigma\left(\hat{\mathbf{n}}, \hat{\mathbf{e}}_{d e t}\right)$ coincides with Eq. (7) in Ref. [38].

In sections 6 and 7 of this paper we explore the internal electric fields of different particles, and look at their scattering properties. We plot $\sigma$ on polar plots as a function of $\hat{\mathbf{n}}$, for both $\hat{\mathbf{n}}$ and $\hat{\mathbf{e}}_{d e t}$ in the $x-y$ plane.

\subsection{Accuracy of the DDA method}

Two conditions are specified by Draine and Flatau [35] to minimize errors and ensure that the DDA formulation is valid:

1. The dipole spacing $d$ must be sufficiently small compared to the internal wavelength of the particle. The condition given in their study is that the number of dipoles per internal wavelength, $n_{\lambda}$, should exceed a value of $2 \pi$. Zubko et al. [39] showed that the DDA provides highly accurate results for irregular particles with this condition. However, a more restrictive value of $n_{\lambda}>4 \pi$ is recommended by Draine and Flatau for scattering phase function calculations such as radar cross sections [40]. This value has been employed in some scattering studies, e.g. Ref. [18]. The most commonly used convention for discretisation is to prescribe at least 10 dipoles per internal wavelength, i.e. $n_{\lambda} \geqslant 10$. Yurkin and Hoekstra [37] state that this constraint is a good first guess for many applications, but accuracy is not guaranteed, particularly for large size parameters. Comparisons with Mie theory for solid ice spheres have shown that in fact $n_{\lambda}>42$ may be required for accurate values of $\sigma_{\text {back }}$ [38].

2. The shape of the particle must be described adequately by ensuring that $N$ is sufficiently large, i.e. $d$ is small enough for the results to converge. It is unclear from the literature how to quantify this condition, so the required $N$ is calculated on a case-by-case basis. See section 5.2 for further details.

\section{Validation of the numerical method}

\subsection{Verification of DDA internal field results using BEM++}

As discussed in section 4.1, the accuracy of the DDA method is sensitive to both discretisation and shape errors. A detailed summary of previous attempts to quantify these errors is given by Yurkin and Hoekstra [37].

In this paper we present results for 2 different size parameters of $x=2$ and $x=10$. To do these calculations, a version of the DDA method was implemented in Matlab. This was done to develop a deeper understanding of the DDA method, to have convenient control over input geometries and visualisation of results, and to have a basis to develop simplified approximate methods. Such simplified methods are described in more detail in Ref. [41]. We note that the code used here is not highly optimised, unlike popular open-source codes such as DDSCAT [35] and ADDA [42].

To verify the implementation and validate the findings presented here, we compared a number of results to those obtained using a fundamentally different numerical method. In the Boundary Element Method (BEM), the electric field is obtained by formulating the problem as boundary integral equations. Groth et al. [43] studied the performance of BEM for the problem of scattering by ice particles, using an open source boundary element library, BEM++, developed by Śmigaj et al. [44]. In that paper they show that by using 10 mesh elements per wavelength, BEM++ gives results for scattering and extinction efficiencies, and phase function that are accurate to within $1 \%$. However, the accuracy of the internal fields was not investigated. The difference between the internal field obtained using our DDA code and the BEM++ setup used by Ref. [43] was calculated as:

$$
\frac{\left\|\mathbf{E}_{D D A}-\mathbf{E}_{B E M}\right\|}{\left\|\mathbf{E}_{B E M}\right\|} \times 100 \%
$$


where $\mathbf{E}_{D D A, j}$ and $\mathbf{E}_{B E M, j}$ are the solutions evaluated in the centre of the $j$ th dipole, using DDA and BEM++ respectively.

This experiment was performed for a thin hexagonal plate of solid ice, with a size parameter of 2 and an aspect ratio of 0.1 . For the calculations we use 17 elements per wavelength for $\mathrm{BEM}++$, which is better than the required $\mathrm{BEM}++$ resolution specified in Ref. [43] . The difference from Eq. (9) was calculated using different grid refinements for DDA. The dipole size was determined by specifying the number of dipoles along the smallest dimension of the particle. In the case of the thin plate, this means we specify the number in the vertical dimension $\left(n_{z}\right)$. The difference obtained for various values of $n_{z}$, and their corresponding number of dipoles per internal wavelength, $n_{\lambda}$, was calculated. The results ranged from $1.2 \%$ for $n_{z}=3$ $\left(n_{\lambda}=26\right)$, to $0.6 \%$ for $n_{z}=7\left(n_{\lambda}=61\right)$. This confirms that the DDA code is performing as expected, and thus we are confident that it can be used for the $x=2$ calculations in this study.

Unfortunately we could not perform analogous comparisons for $x=10$, as the resources we had available were insufficient to run $\mathrm{BEM}++$ for that case. Therefore, we took an alternative approach to examine the accuracy of DDA calculations for $x=$ 10 , outlined in the following section.

\subsection{Convergence of DDA internal field results with increasing} $n_{\lambda}$

In this section we explore how the internal field calculations for $x=10$ converge as the number of dipoles per internal wavelength is increased.

Fig. 1 examines the convergence of the DDA internal field with increased grid resolution. The lines show the real part of the $x$ component of the field though the centre of a hexagonal plate of $x=10$ and aspect ratio 0.1 . The results for different grid refinements of $n_{z}=10,13,15$, and 17 have been plotted in the figure, corresponding to $n_{\lambda}=17,23,26$, and 30 . The curves are qualitatively similar for each of the different grid resolutions, with the main differences occurring in the field amplitudes.

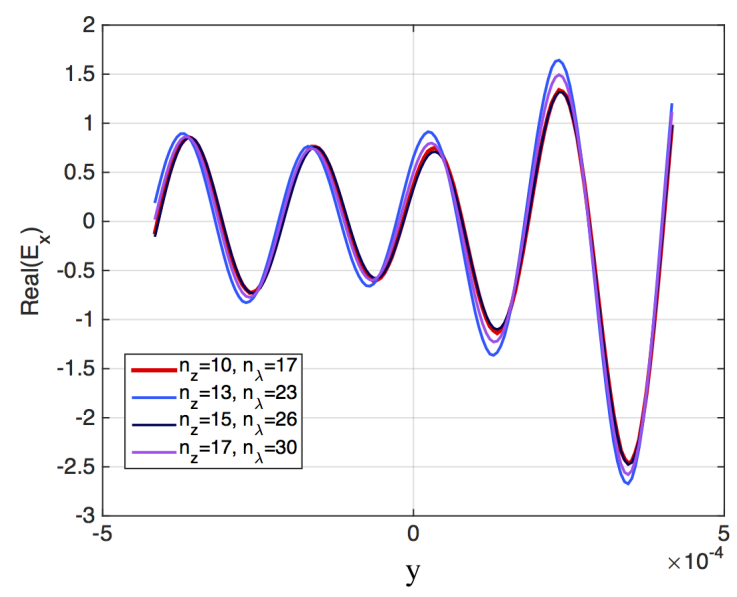

Figure 1: Real $\left(E_{x}\right)$ through the centre of a hexagonal plate of $x=10$ and aspect ratio 0.1 . The colours show different grid refinements of $n_{z}=10,13,15,17$, corresponding to $n_{\lambda}=17,23,26,30$.
We estimate the error in the internal fields by comparing our highest resolution case of $n_{\lambda}=30$ to results of lower resolution, ranging from $n_{\lambda}=8$ to 26 . The calculations are done in an analogous manner to Eq. (9). We find that using a minimum of 17 dipoles per internal wavelength gives an error below $10 \%$ in the field for $x=10$.

This experiment was also done for $x=2$. It is noted that using the same discretisation for $x=2$ results in higher values of $n_{\lambda}$, so greater accuracy is expected. The values tested range between $n_{\lambda}=44$ and 124, uncovering much smaller errors below $0.5 \%$. The accuracy estimated via these tests is sufficient for exploring the qualitative properties we are interested in here, i.e. the variation of internal field with particle size and shape, and the effects of these changes on far-field scattering.

It is worth noting that despite the fact we are well within the commonly prescribed criterion of $n_{\lambda}=10$, convergence with increasing resolution is slow. Although the fields are very similar, the results in some regions of the particle have not completely converged, and the relative errors do not decrease monotonically as $n_{\lambda}$ is increased. Yurkin et al. [45] found a similar pattern, highlighting that the issue only occurs with shapes that cannot be modelled exactly by cubical cells. Inaccurate representation of boundaries leads to small variations in particle shape with discretisation, which in turn causes oscillating errors. However, this does not affect the conclusions we are drawing in the rest of the paper.

Comparisons of the corresponding scattering cross section results for these discretisations (not shown for brevity) reveals that the error in the far field is mainly concentrated in the backward direction. Backscatter is very sensitive to particle discretisation, whereas scattering in other directions shows little variation with discretisation. This result is consistent with Petty and Huang [38] who show that smaller dipole spacing is required for accurate backscatter results. As pointed out in the previous section, they suggest using a value of $n_{\lambda}>42$ for accurate $\sigma_{\text {back }}$ in the case of ice spheres, and with our highest resolution we are only using $n_{\lambda}=30$. It is possible that a finer discretisation may be needed if accurate backscatter calculations are required.

\section{Results and discussion - Pristine monocrystals}

\subsection{Hexagonal plate}

\subsubsection{Internal field}

Fig. 2 shows the magnitude of the internal electric field through the central horizontal plane of a hexagonal plate of solid ice, with a maximum dimension $\left(D_{\max }\right)$ of $1 \mathrm{~mm}$ and an aspect ratio of 0.1 . The plate is discretised with $n_{z}=15$, resulting in approximately $n_{\lambda}=132$ and $n_{\lambda}=26$ for $x=2$ and $x=10$ respectively. The incident plane wave propagates in the $y$ direction, i.e. from the top of the page to the bottom, and is polarised along the $x$-axis. Note that the color scales in Figs. $2 \mathrm{a}$ and $2 \mathrm{~b}$ are not the same. The plots shown for $x=2$ in this manuscript use a smaller amplitude range than that used in their $x=10$ counterparts, in order to show some detail for the smaller size parameter. This is the case for the majority of the 


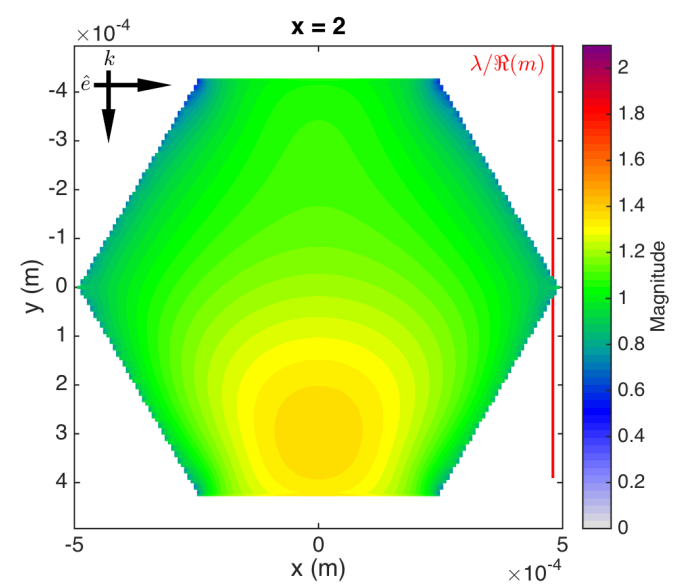

(a)

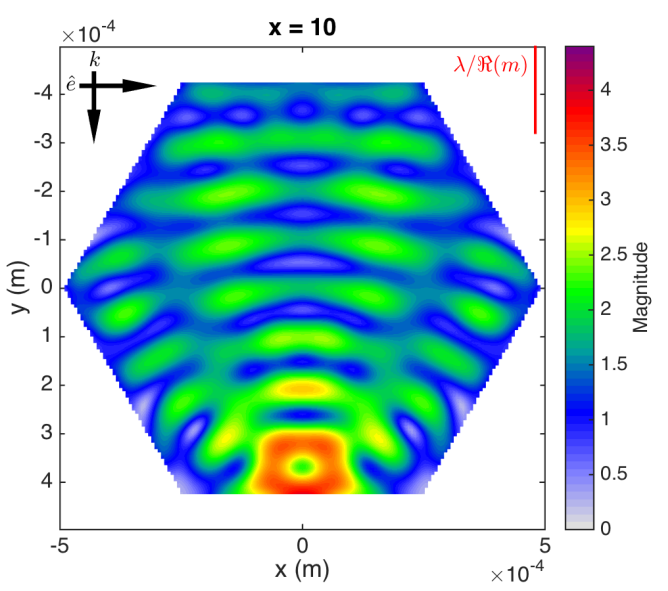

(b)

Figure 2: Magnitude of the internal field through the central horizontal plane of a hexagonal plate of aspect ratio 0.1 for $(a) x=2$ and $(b) x=10$. The arrows show the direction of propagation and polarisation, which are perpendicular to each other in the $x-y$ plane. The particle is discretised with $n_{z}=15$, resulting in $(a) n_{\lambda}=132 ;(b) n_{\lambda}=26$.

internal field magnitude plots presented here, with the exception of the complex aggregate in Fig. 14.

The average and peak values of the field for both size parameters are given in Table 1, along with results for a smaller value of $x=0.01$. For very small values of $x \ll 1$, particles are in the Rayleigh scattering limit. In this size regime, the internal field has approximately constant magnitude, with the maximum and average values varying by only a factor of 1.15 . For $x=2$ and $x=10$, the largest value of electric field is observed close to the perimeter of the particle, at the opposite side from where the wave hits the plate. We refer to this as the forward region of the particle. For $x=2$ there is a rather broad maximum centred at approximately $0.1 \mathrm{~mm}$ from the forward edge of the plate, with the peak magnitude reaching 1.3744. For $x=10$, the focussing behaviour becomes more obvious, and the maximum magnitude also increases. There is an "O" shaped region where the field magnitude is largest, reaching a value close to 4 . Supplementary plots S1-S3 show how the field changes within the hexagonal plate geometry for intermediate size parameters between 2 and 10. The focussing pattern is a persistent feature, becoming more prominent with increased $x$. Therefore, this is not a resonant phenomenon. The maximum magnitude within the particle broadly increases with $x$. It is interesting to note that for all size parameters shown, the magnitudes are considerably higher than the value of $\left|\mathbf{E}_{j}\right|=\left|\mathbf{E}_{j}^{i n c} \alpha_{j} / V_{j} \chi_{j}\right| \approx 0.58$ that would result from the presence of the applied wave only. Hence there is a strong coupling between the dipoles across the crystal, and RGA provides a poor approximation to the field in this case.

Another interesting observation is that the inhomogeneity along the $x$-axis in Fig. 2b resembles diffraction and interference patterns, such as those resulting from Young's double-slit experiments. This suggests that we are entering a regime where physical optics approximations could be used. Geometric optics may be applied for size parameters much larger than the incident wavelength. However, improvements to geometric optics methods have been shown to be accurate for $x$ as small as 18 , e.g. using methods such as ray tracing with diffraction on facets (RTDF; [46]). Hence it is possible that for the larger size parameters considered here, physical or geometric optics methods may be suitable approximations to apply.

The concentration of the electric field is similar to the focussing nature of a convex optical lens, and is caused by a change in wavelength, due to $m$, that takes place inside the particle. The difference in wavelength appears slightly more pronounced through the centre of the particle than it does close to the boundaries, resulting in a curved wave front which focusses the field towards one side of the plate. Note that the symmetric structure and focussing behaviour of the field also occurs if the incident wave is propagating in the $x$ direction, such that it encounters a corner of the plate rather than a flat side. Similarly, the symmetry and focussing remain when the wave is polarised orthogonal to the direction shown here, i.e. in the $z$ direction. These results can be seen in Figs. S4 and S5 of the supplementary material. However, for the $z$ polarised wave, the focussing occurs over a smaller region and is less prominent than in the $x$ polarised case, showing a maximum magnitude that is $23 \%$ lower.

It is interesting to do some experiments to explore whether the phenomena above are a special case resulting from the symmetry of the setup, or whether they are part of a more general behaviour. We divide our sensitivity tests into 2 categories:

1. Experiments with $\hat{\mathbf{k}}$ in the $x-y$ plane but not along an axis of symmetry of the hexagon.

2. Experiments where $\hat{\mathbf{k}}$ is rotated around the $\mathrm{x}$-axis to lie at an angle in the $y-z$ plane.

Fig. S6 in the supplementary material shows the internal field when the incident wave is directed at an offset of $20^{\circ}$ from the positive $y$-axis in the $x-y$ plane. In this case the focussing behaviour is still prominent, but the symmetry of the field is lost as the incident wave is no longer directed along a particle axis of symmetry.

Figs. S7 - S9 of the supplementary material show the internal field magnitudes for incident angles of $20^{\circ}, 70^{\circ}$, and $90^{\circ}$ in the 
$y-z$ plane. Focussing behaviour is still found for the smallest angle of $20^{\circ}$ in the $y-z$ plane (Fig. S7), but the location of the focussing has moved slightly. There is a region at the bottom of the plate towards the forward side of the particle where the field values are large. An apparent internal reflection from the lower basal face results in the high field values being redirected towards the top of the particle. The field corresponding to this incident direction sees a decrease in maximum magnitude by approximately $10 \%$, when compared to the case in Fig. 2b. Increasing the incident angle to $70^{\circ}$ in the $y$ - $z$ plane diminishes much of the focussing behaviour. In this case the maximum magnitude decreases to a value approximately $50 \%$ lower than in Fig. 2b. Further increasing the angle to $90^{\circ}$, i.e. directing the incident wave from above a basal face, the maximum magnitude is found to occur close to the centre of the particle. Although the maximum is slightly larger than at $70^{\circ}$, it is still $42 \%$ lower than the horizontally directed case. Thus the largest fields are found when the incident wave is directed in a more horizontal direction, hitting the sides of the particle rather than the basal faces, even if the incident angle is not directed along an axis of symmetry. Lower field magnitudes result when the effective size parameter in the direction of propagation is small, as is the case for the incident wave directed at $70^{\circ}$ and $90^{\circ}$ in the $y-z$ plane.

We explore the individual components of the field shown in Fig. 2b, where the incident wave is directed in the $y$ direction and polarised in the $x$ direction. Fig. 3 shows the real part of the components of the field which are perpendicular and parallel to the direction of propagation, i.e. the $x$ and $y$ components, for $x=10$. This is what Tyynelä et al. [18] refer to as the transverse and longitudinal components of the field. The real part of the $z$ component is approximately $10^{-5}$ in the central plane of the particle. It has a maximum of 0.55 on the top and bottom layers, and globally in $L^{2}$-norm $E_{z}$ is 20 times smaller than $E_{x}$. For that reason, the $z$ component is not shown here, but the reader is directed to Fig. S10 of the supplementary material if interested. Unlike the $z$ component, the $x$ and $y$ components do not change much across the different layers within the plate. The fields are slightly larger in the central plane than on the top and bottom layers for the $x$ and $y$ components. For both $E_{x}$ and $E_{y}$, the field through the middle layer is approximately $30 \%$ larger than on the top and bottom layers. For $E_{z}$, the top and bottom layers have values which are $10^{4}$ times larger than in the middle layer. The structure is concentrated on the particle boundary for $E_{z}$.

It is clear that the total field in Fig. $2 b$ is a combination of two distinct waves. The $x$ component in Fig. 3a shows a curved wave front extending through the bulk of the particle. The $y$ component in Fig. $3 b$ has a different structure resembling a standing wave around the perimeter of the plate, comprising a series of nodes (minima) and antinodes (maxima). It is seen in Fig. 3a that $\mathfrak{R}\left(E_{x}\right)$ has even symmetry, whereas the plot of $\mathfrak{R}\left(E_{y}\right)$ in Fig. 3b has odd symmetry. The focussing of a planelike wave through the bulk of the crystal, plus a standing wave close to the boundary is similar to the behaviour found in spherical and cylindrical particles larger than the wavelength in Refs. $[16,17,47]$. As mentioned, we see this behaviour over a range

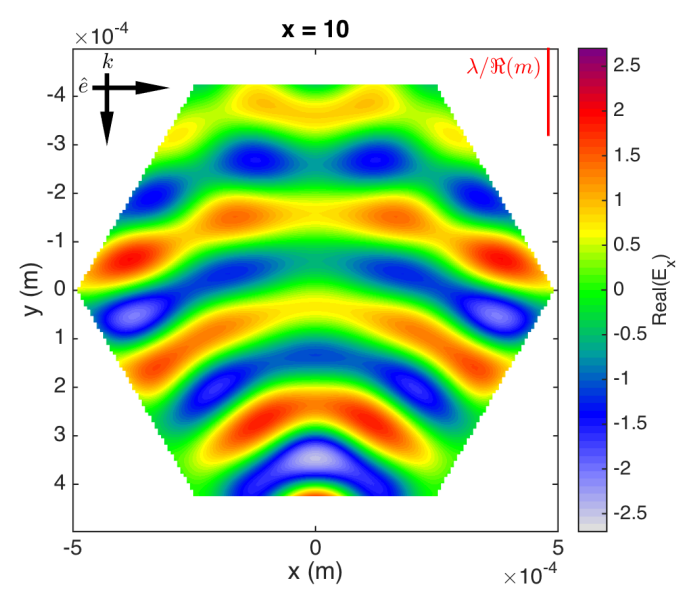

(a)

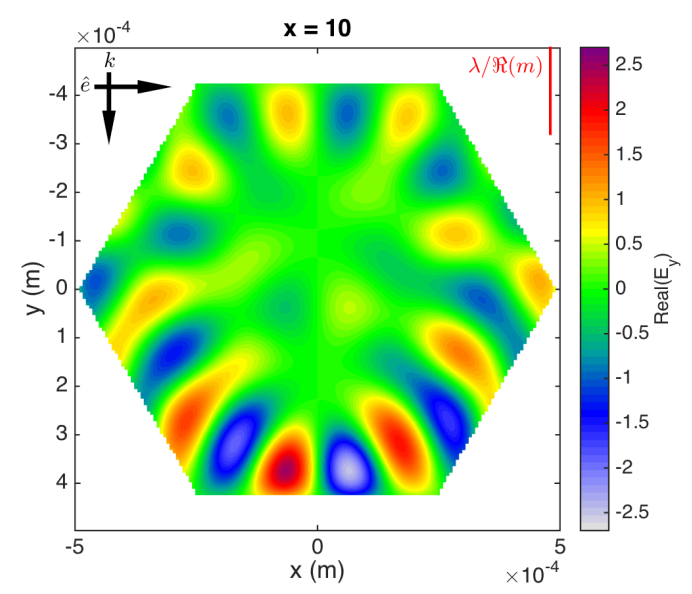

(b)

Figure 3: Real part of the (a) $x$ component and (b) $y$ component of the internal electric field for $x=10$.

of different values of $x$ in the hexagonal plate, showing that this is an "off-resonance" phenomenon. Similar wave structures are observed for all size parameters, though it is less clear that a standing wave is present around the perimeter for smaller size parameters as there are fewer nodes and antinodes in these cases. Resonances may well exist at very specific values of $x$, however we have not found them, and unlike the case of spheres and infinite cylinders, there is no simple way to accurately predict the size parameters at which they would occur.

The different components of the field within the hexagonal plate of $x=10$ for a $z$ polarised wave have also been examined (not shown for brevity). $\mathfrak{R}\left(E_{x}\right)$ and $\mathfrak{R}\left(E_{y}\right)$ display fields with a node in the central plane of the particle, oscillating in sign between the top and bottom layers. $\mathfrak{R}\left(E_{z}\right)$ displays minimum values at the particle surface and reaches a maximum in the central plane, while the sign does not oscillate throughout the particle.

As expected, the wave extending through the particle in Fig. 3a has a shorter wavelength than in the exterior medium due to the refractive index, $m$, of the particle. For wavelengthscale spherical particles, analogous studies by Refs. [48, 49] 
found the wavelength through the central plane to be approximately equal to the material wavelength, i.e. $\lambda / \mathfrak{R}(m)$. In our case of a hexagonal plate with a wavelength smaller than the particle size, we have estimated that the external wavelength exceeds that inside the plate by a factor of $1.5 \pm 0.2$. This is slightly lower than the factor of approximately 1.78 that would result if the wavelength was dictated by the refractive index alone. This may be because we are looking at a flat geometry where even the central points are close to the particle boundary, so we don't see the material wavelength.

The standing wave around the perimeter has a longer wavelength that is more comparable to that of the incident wave, since it is located very close to the boundary. In this case, we have estimated that the incident wavelength is approximately $1.3 \pm 0.2$ times that of the standing wave. Again, this behaviour was observed in spherical particles by Refs. [48, 49], who also found that the number of maxima or minima around the perimeter was equal to the value of $x$. However, in our case we have found the number of maxima and minima to be less than $x$, counting a total of 9 each in Fig. $3 b$.

\subsubsection{Far-field scattering}

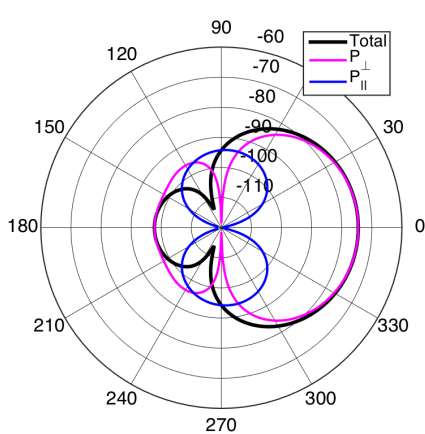

(a) $x=2$

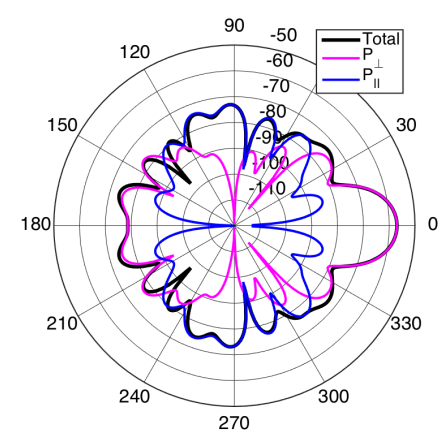

(b) $x=10$
Figure 4: Polar plots of the differential scattering cross section of a hexagonal plate as a function of the propagation $\hat{\mathbf{n}}$ and polarisation $\hat{\mathbf{e}}_{d e t}$ directions of the scattered wave. This is calculated using all components of the field (black), only the perpendicular component $\left(P_{\perp}\right.$; magenta), and only the parallel component $\left(P_{\|} ;\right.$blue). The value at $0^{\circ}$ represents forward scattering, and $180^{\circ}$ is backscatter.

It is interesting to explore the effect the two different wave structures in Fig. 3 have on far-field scattering. To do this, Eq. (8) was used to calculate the differential scattering cross section, $\sigma$, computed at scattering angle intervals of $1^{\circ}$. These calculations are displayed on a polar plot using $\mathrm{dB}$, i.e. $10 \log _{10}(\sigma)$. Forward scattering is located at $0^{\circ}$ on the plot, and backscatter is shown at $180^{\circ}$. It is noted that for $x=0.01$ (not shown), scattering at different angles is less sensitive to particle shape, and scattering in the forward and backward directions is approximately equal. The cross section for $x=2$ and $x=10$ has been computed in 3 different ways: $(i)$ using all components of the internal field; (ii) using only the component perpendicular to the incident wave $\left(P_{\perp}\right)$; and (iii) using only the component parallel to the incident wave $\left(P_{\|}\right)$. These correspond to the $x$ and $y$ components in this case.

Fig. 4 shows the result for the hexagonal plate of $x=2$ and $x=10$. In both cases it can be seen that the transverse component, $P_{\perp}$, contributes more to scattering near the forward and backward directions, with a smaller contribution from $P_{\perp}$ at intermediate scattering angles close to $90^{\circ}$ and $270^{\circ}$. Conversely, $P_{\|}$contributes more to the total scattering at these angles, and less in the forward and backward directions. This is expected from Eq. (8), as the polarisation direction $\hat{\mathbf{e}}_{d e t}$ is perpendicular to the observation direction $\hat{\mathbf{n}}$. It is interesting to note that for $x=0.01, P_{\perp}$ is responsible for the total differential scattering cross section, and no contribution from $P_{\|}$is apparent at any angle. Contributions from the parallel component emerge as the size parameter is increased to approximately $x>1$. We deduce that for the case shown here, the standing wave structure around the perimeter of the hexagonal plate contributes predominantly to sidescattering, and the wave extending through the centre is responsible for scattering at angles proximate to the forward and backward directions.

The differential scattering cross section for the $z$ polarised incident wave is also explored, this time plotting the results in the $y-z$ plane. Interested readers can find this in Fig. S11 of the supplementary material. The same conclusions are drawn from this scenario - the transverse component (corresponding to the $z$ component in this case) dominates forward and backward scattering, and the component in the direction of propagation $\left(P_{y}\right)$ contributes mainly to sidescatter.

The results presented here help us to understand what controls the asymmetry parameter, $g$, which describes how much incident radiation is scattered in the forward and backward directions. Therefore, the findings could be useful for developing parameterisations of $g$ for use in radiative transfer simulations.

\subsection{Cylindrical disk and spheroid}

Realistic ice particles have historically been approximated by simpler shapes such as spheres and spheroids in order to calculate their scattering properties. Much of the literature has shown that this method produces poor results for particles outside the Rayleigh regime [50]. It is interesting to explore whether it is possible to gain greater physical insight into why these methods fail to produce accurate results by modelling different approximations to the plate.

The magnitude of the internal field is plotted for a cylindrical disk of solid ice, with aspect ratio and $D_{\max }$ equal to those of the hexagonal plate in section 6.1. This allows us to look at the effect of particle shape on the structure of the field. We also look at a solid ice spheroid of equal aspect ratio and $D_{\max }$. Figs. 5 and 6 show the results for $x=2$ and $x=10$, respectively. The real parts of the $x$ and $y$ components of the field for $x=10$ can be seen in Fig. S12 of the supplementary material. Overall, there are clear similarities between the structure of the field within both of these particle shapes, compared to the hexagonal plate. The same phenomenology applies to both geometries, and the details of the perimeter of the particle are not critical to produce similar internal fields. The field is more uniform 


\begin{tabular}{c|cc|cc|cc} 
& $x=0.01$ & & $x=2$ & & $x=10$ & \\
\hline Geometry & Avg. $|\mathbf{E}|$ & Max $|\mathbf{E}|$ & Avg. $|\mathbf{E}|$ & Max $|\mathbf{E}|$ & Avg. $|\mathbf{E}|$ & Max $|\mathbf{E}|$ \\
\hline 1 hex plate & 0.8169 & 0.9414 & 1.0969 & 1.3744 & 1.4108 & 3.9786 \\
2 hex plates & 0.8136 & 0.9173 & 0.9464 & 1.0949 & 1.6021 & 3.0049 \\
5 hex plates & 0.8583 & 1.2337 & 0.9111 & 1.3866 & 1.5049 & 2.3575 \\
Aggregate 1 & 0.6503 & 1.2430 & 0.6593 & 1.2617 & 0.6986 & 1.4302 \\
Aggregate 5 & 0.6593 & 1.2097 & 0.6650 & 1.2212 & 0.6879 & 1.2400
\end{tabular}

Table 1: Average and maximum internal field magnitudes for different geometries of $x=0.01, x=2$ and $x=10$. The geometries included are the single hexagonal plate, aggregates of 2 and 5 plates, and 2 different arrangements of 10 "fernlike dendrite" monomers modelled by Tyynelä et al. [30] . "Aggregate 1 " is the particle shown in Fig. 14; "Aggregate 5 " is not shown for brevity. For reference, $|\mathbf{E}| \approx 0.58$ in the presence of the applied wave only.

for $x=2$, increasing in complexity for $x=10$. The focussing behaviour in the forward region is also prominent for both the disk and spheroid. For $x=2$, a slightly smaller amount of focussing is seen in the spheroid than in the disk and the hexagonal plate. Conversely, for $x=10$, the spheroid exhibits more focussing than the other geometries. Within the spheroidal geometry, the wave crests (in green) appear to be more curved, extending to the the particle edge. This could be because the spheroidal shape is very thin close to the perimeter, resulting in less interference from different layers in these areas.

The structure of the fields within the cylindrical disk and the hexagonal plate exhibit clear resemblances, suggesting that in terms of approximations for a hexagonal geometry, a disk may provide superior results to a spheroid. However, in terms of the average magnitudes, the spheroid displays results that are closer to the plate, giving values within $1 \%$ for $x=2$, and $3 \%$ for $x=10$. The average values for the disk differ from the plate by approximately $4 \%$ and $13 \%$ for $x=2$ and $x=10$. For $x=2$, the maximum magnitudes of the disk and spheroid are within $3 \%$ and $4 \%$ of the plate, respectively. For the larger size parameter of $x=10$, both geometries have maximum values that differ from the plate by about $10 \%$, but the spheroidal shape results in an overestimation while the disk gives an underestimation.

\subsection{Hexagonal column}

\subsubsection{Internal field}

Fig. 7 shows the field inside a hexagonal prism of aspect ratio 1 for $x=2$ and $x=10$. Slices have been plotted through the planes $x=0 \mathrm{~m}$ and $z=-1.2 \times 10^{-4} \mathrm{~m}$, where $|\mathbf{E}|$ is at a maximum for $x=10$. The particle has the same value of $D_{\max }$ as the plate. It can be seen that by increasing the aspect ratio, more focussing is seen for $x=2$ compared to the plate, and the maximum field strength is approximately $20 \%$ larger. For $x=10$, Fig. $7 \mathrm{~b}$ shows that the increased aspect ratio results in 2 primary regions of focussing, but the maximum value of these is smaller than in the flat plate. There appears to be more destructive interference in the prism, with the average magnitude in the plate exceeding that in the prism by a factor of 1.3. Investigating the real part of the $x$ and $y$ components of the field for $x=10$ shows that these are qualitatively very similar to the field components of the plate (not shown for brevity). The transverse component for the column exhibits a wave extending though the particle, and the component in the direction of propagation displays a well defined standing wave structure around the perimeter.

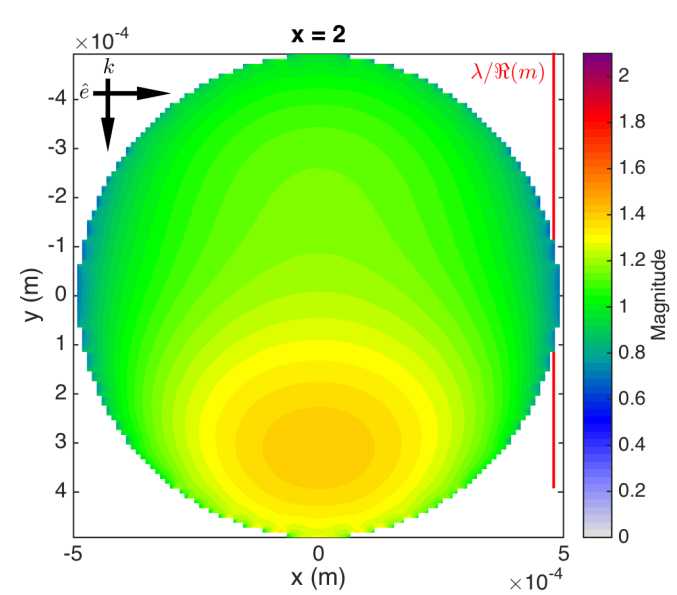

(a) Disk

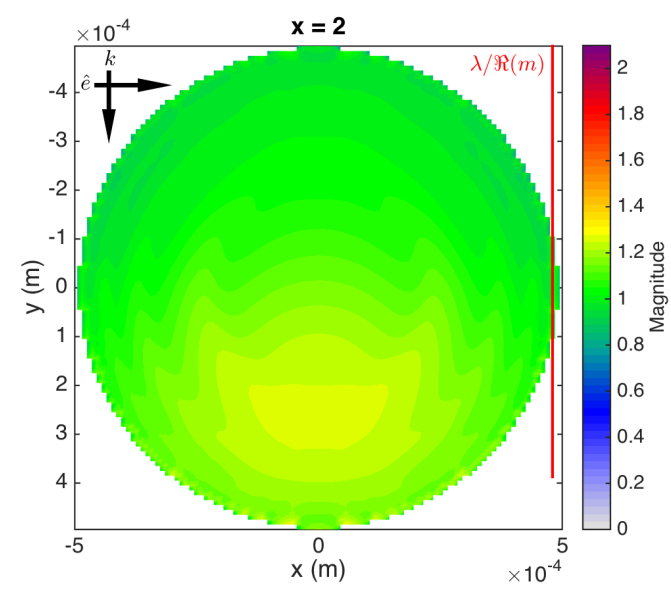

(b) Spheroid

Figure 5: Magnitude of the internal field of $(a)$ a disk and $(b)$ a spheroid of $x=2$. Both particles have the same aspect ratio and $D_{\max }$ as the hexagonal plate in section 6.1 .

Fig. S13 in the supplementary material shows the case equivalent to Fig. $7 b$, but with the incident wave along the $z$-axis, hitting the top basal face of the prism. This corresponds to an angle of $90^{\circ}$, as discussed for the plate in section 6.1. For the thin plate, it was found that this setup results in an internal field with lower maximum magnitudes than when the incident wave is directed in the $x-y$ plane. For the prism of aspect ratio 1, this 


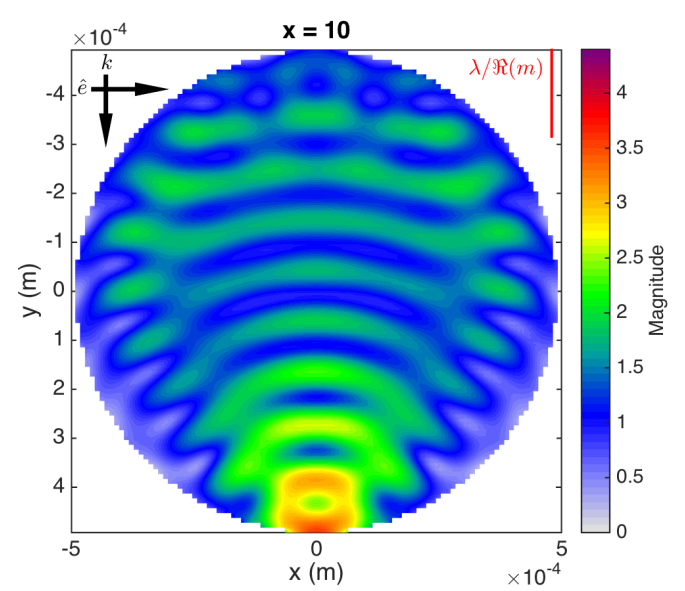

(a) Disk

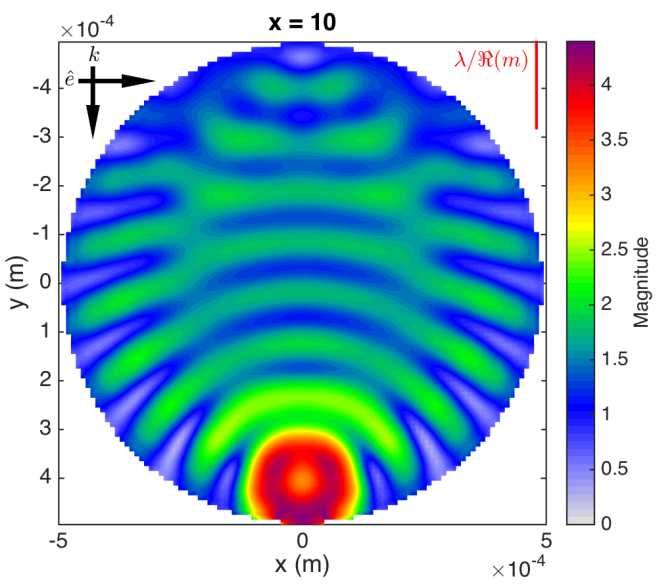

(b) Spheroid

Figure 6: As in Fig. 5 but for $x=10$.

is not the case. Strong focussing behaviour is seen towards the forward region of the particle. Thus, the lower field value found for the plate when the incident angle is directed at $90^{\circ}$ is a result of the aspect ratio of the particle. The effective size parameter in the direction of propagation is small for the thin plate, resulting in a diminished field, but this is not the case for the thick prism.

\subsubsection{Far-field scattering}

Polar plots of $\sigma$ are displayed in Fig. 8 for a hexagonal column, to explore the effect of aspect ratio on the differential scattering cross section. The overall pattern resembles that of the thin plate with qualitatively similar $P_{\perp}$ and $P_{\|}$contributions. Quantitatively, there are some large differences, particularly in the backscatter direction for $x=10$. However, as mentioned in section 5.2, backscatter is very sensitive to discretisation. Therefore a finer mesh resolution would be required in order to make meaningful conclusions about changes in $\sigma_{\text {back }}$ with aspect ratio.

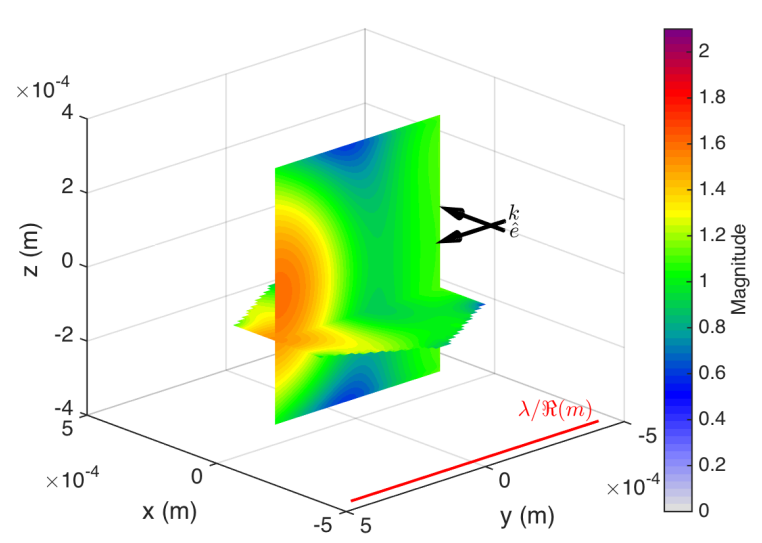

(a) $x=2$

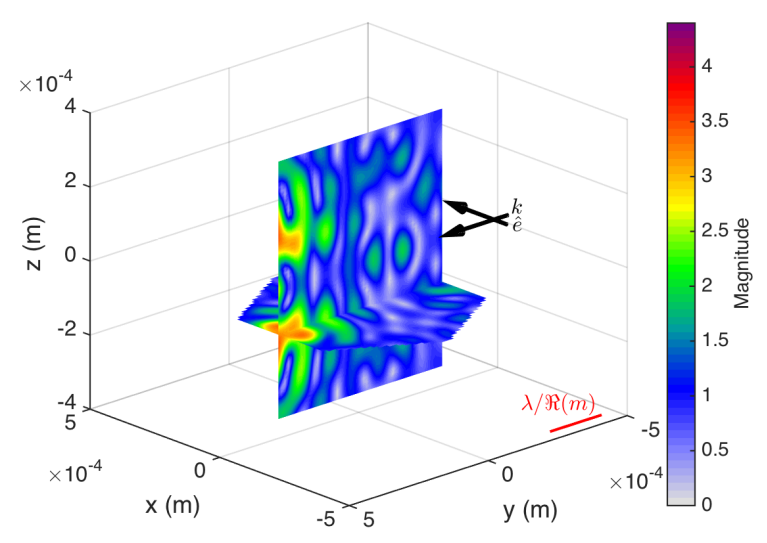

(b) $x=10$

Figure 7: Magnitude of the internal field of a hexagonal column of aspect ratio 1 of $(a) x=2 ;(b) x=10$. Slices are shown through $x=0 \mathrm{~m}$ and $z=-1.2 \times 10^{-4} \mathrm{~m}$. These particles have a value of $n_{\lambda}=62$ and 17 , respectively.
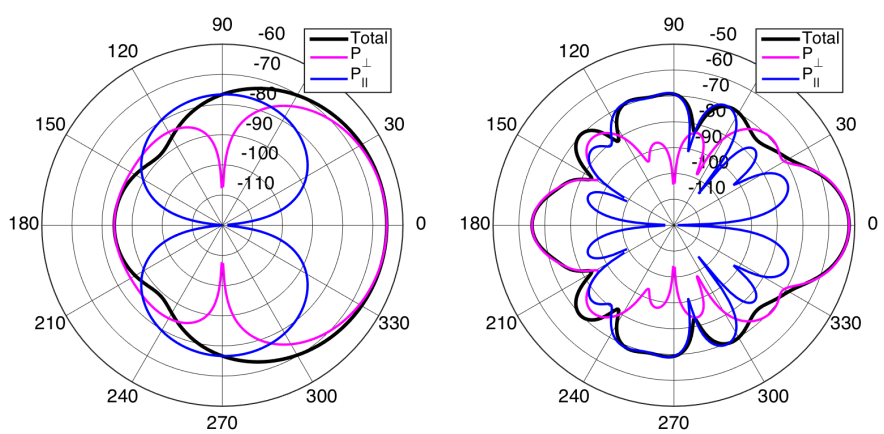

(a) $x=2$

(b) $x=10$

Figure 8: Differential scattering cross section, as in Fig. 4, but for a hexagonal prism of aspect ratio 1 .

\subsection{Cylindrical column and sphere of same $D_{\max }$ as hexagonal prism}

In section 6.2 we explored the differences between the inter10 


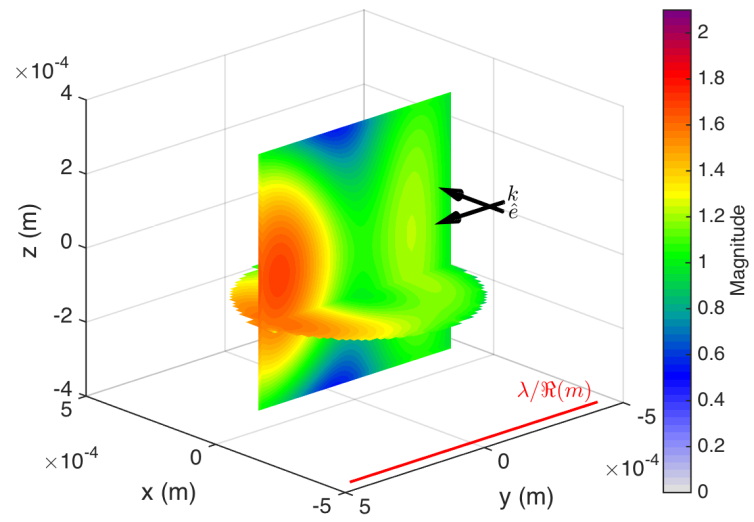

(a)

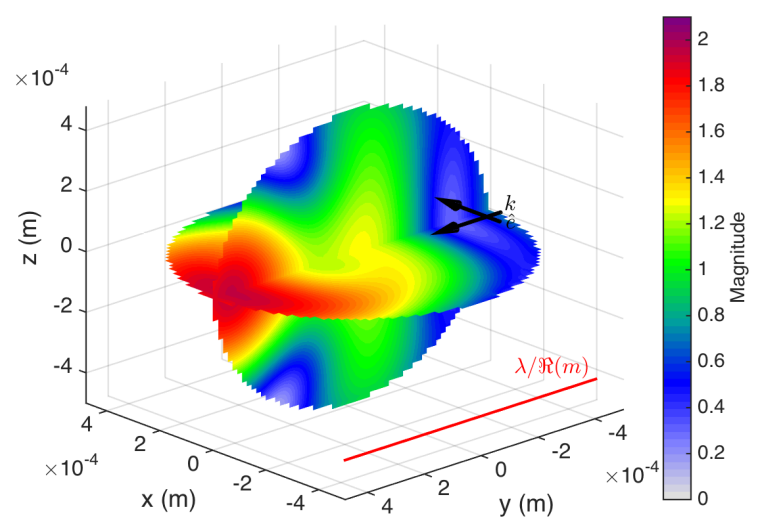

(b)

Figure 9: Magnitude of the internal field of $(a)$ a cylindrical column and $(b)$ a sphere of aspect ratio 1 for $x=2$.

and spheroid. Analogous to this, we have compared the taller hexagonal prism to a sphere and right circular cylinder of equivalent aspect ratio and $D_{\max }$. Figs. 9 and 10 show the results for $x=2$ and $x=10$. For both values of $x$, it is observed that spheres have one principle region of focussing, rather than the two maxima we observed for the hexagonal column. The cylindrical column bears a closer resemblance to the hexagonal prism geometry in that it displays multiple regions of large magnitude values for $x=10$, although the maximum value is slightly larger for the cylindrical column, exceeding the hexagonal geometry by approximately $6 \%$. However, the maximum magnitude of 6.6 found for the sphere of $x=10$ overestimates the hexagonal case by $75 \%$. It is noted that in order to enable clearer comparisons we fixed the range of the colour scale in Fig. $10 \mathrm{~b}$ to be the same as the other shapes. Although the sphere displays a higher maximum value, the hexagonal and cylindrical prisms have larger average magnitudes than the sphere, by a factor of approximately 1.3. These results show that for the larger aspect ratio of 1 considered here, the cylindrical approximation to the hexagonal particle appears to be superior to the commonly used spherical or spheroidal approximations. In sec-

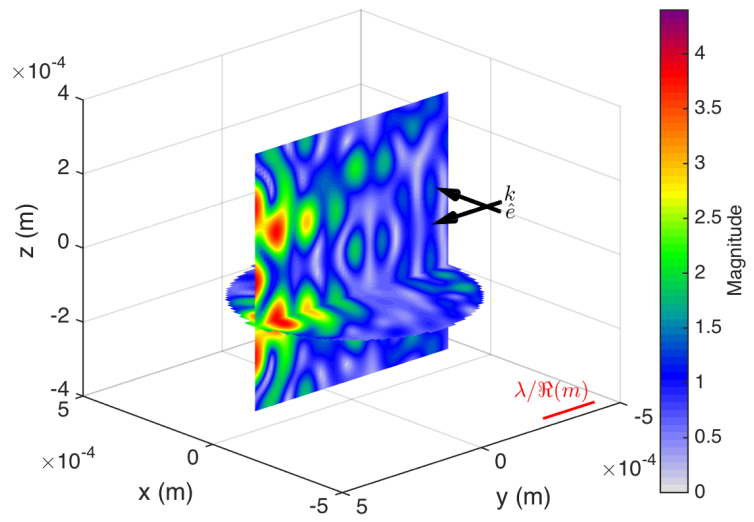

(a)

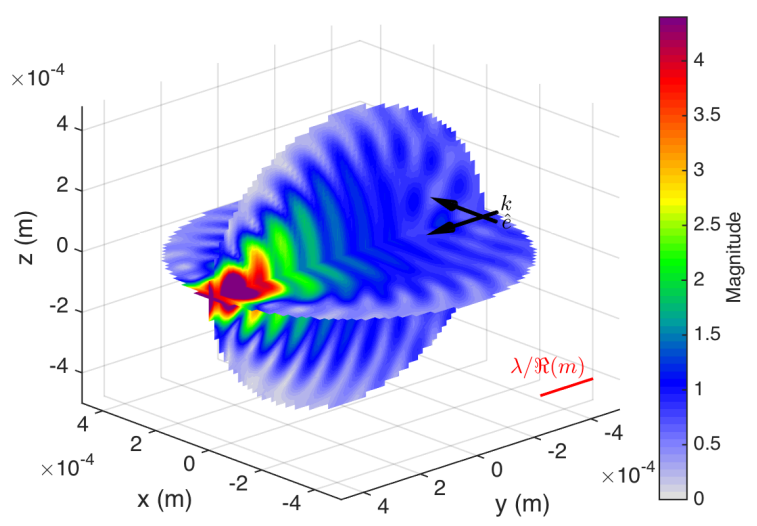

(b)

Figure 10: As in Fig. 9 but for $x=10$.

tion 6.2, it was found that an equivalent aspect ratio spheroid gives a good approximation of the internal field for a smaller aspect ratio of 0.1 . However, it is shown here that as the aspect ratio increases, the approximation becomes less accurate.

\section{Results and discussion - Aggregates}

\subsection{Chain aggregates of plates 1: 2 hexagonal plates}

In the presence of an electric field, chain aggregates of plates can form. Connolly et al. [28] observed that these geometries form predominantly with the prism faces of individual crystals touching. Here we have studied two different aggregates of plates. The first is a simple aggregate of 2 plates, and the second is an aggregate of 5 plates. Both geometries are aligned with prism faces touching, and were generated by Ref. [51] using a stochastic algorithm.

\subsubsection{Internal Field}

First we look at an aggregate of 2 plate-like particles, where each monomer is the same height and $D_{\max }$ as the individual plate studied in section 6.1. Fig. 11 shows that the aggregates 


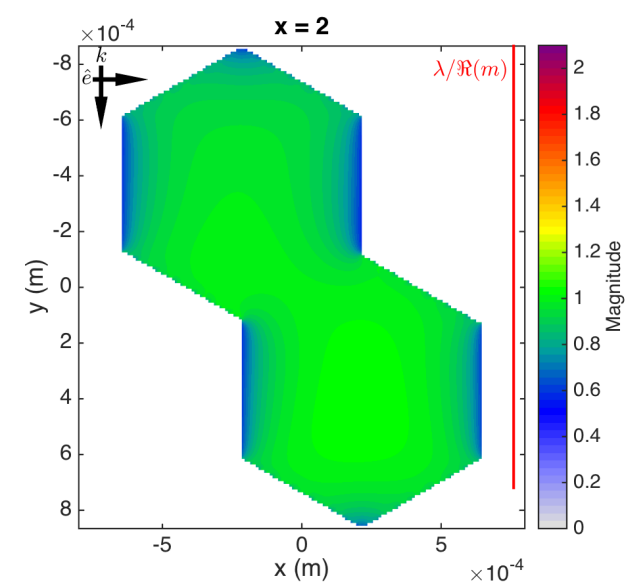

(a) $x=2$

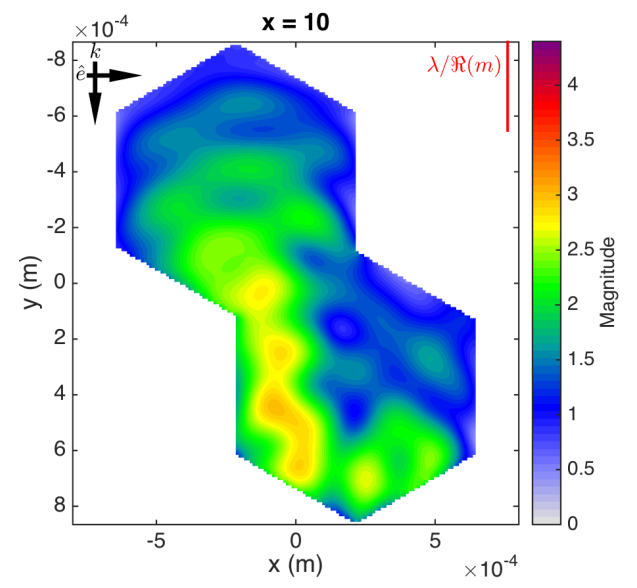

(b) $x=10$

Figure 11: Magnitude of the internal field through the central plane of an aggregate of 2 hexagonal plates for $(a) x=2$ and $(b) x=10$. The number of dipoles per wavelength used in the calculations for these particles is $n_{\lambda}=159$ and 31 , respectively.

of 2 plates exhibit less defined regions of focussing with smaller maximum magnitudes than the previous geometries. For $x=2$, there is very little focussing. In Fig. 11b, it can immediately be seen that for $x=10$ interference between the two plates obfuscates the clear wavy structure and symmetry observed in the hexagonal, cylindrical, and spheroidal monocrystals. These interactions and the resulting field depend on the alignment of the 2 plates. If the arrangement is such that the aggregate of 2 plates is symmetric with respect to the incident wave, the internal field is also symmetric, as expected. An example of this can be seen in Fig. S14 of the supplementary material. Although the field has a symmetric structure in this case, the focussing is still less defined and the maximum magnitude is lower than that calculated for the single plate, suggesting that the complexity of the particle is integral to the loss of structure, rather than the alignment or orientation. We are interested in exploring more irregular aggregates which are frequently observed in clouds. The result in Fig. 11b suggests that the internal field within such particles may not exhibit any clear structure. To test this, the field inside an aggregate of 5 plates was examined, followed by the exploration of more complex aggregates in section 7.4.

\subsection{Chain aggregates of plates 2: 5 hexagonal plates}

\subsubsection{Internal Field}

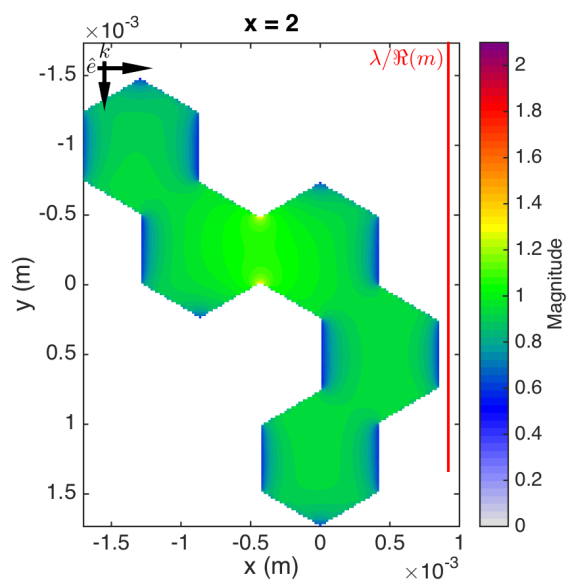

(a) $x=2$

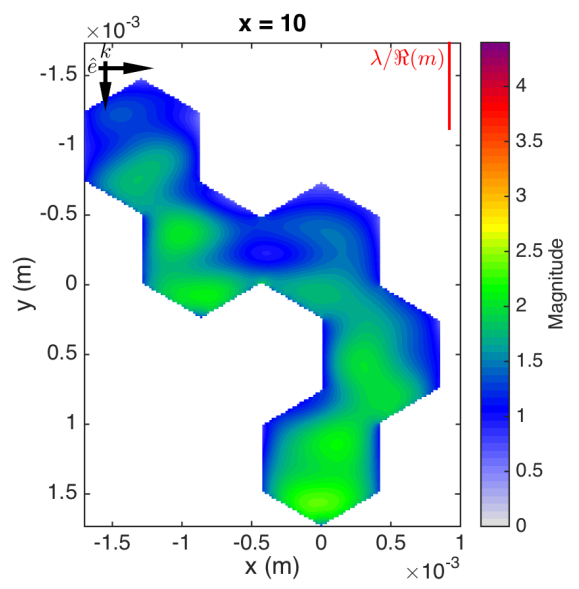

(b) $x=10$

Figure 12: Magnitude of the internal field through the central plane of an aggregate of 5 hexagonal plates for $(a) x=2$ and $(b) x=10$. These particles have a value of $n_{\lambda}=184$ and 36 , respectively.

Fig. 12 shows the field inside an aggregate of 5 hexagonal plates, where each plate has the same dimensions as the particle modelled in Fig. 2. Similarly to what was found for the aggregate of 2 plates, this particle has a field that shows less symmetry than the monocrystals. It is obvious that as the complexity of the geometry increases, there is a clear reduction in magnitude and further dampening of the wavy structure. The internal field magnitude becomes more smoothed and seems to lack the constructive interference that locally changes the magnitude in simpler particles such as the individual plate. However, upon closer inspection, the amount of focussing varies according to the orientation of the particle with respect to the incident wave. For example, directing the incident wave at an angle of $140^{\circ}$ in the clockwise direction from that shown in Fig 12b leads to a maximum magnitude which exceeds that shown here by almost 
$50 \%$. This can be seen in Fig. S15 of the supplementary material. It is noted that this value is still less than that calculated for the single plate. Overall, the focussing effect appears to be more prominent in cases where the path length in the direction of propagation is longer. At $140^{\circ}$, a large amount of focussing is seen as the incident wave is directed at an angle allowing passage through multiple plates with little deviation from the incident direction. This is similar to the results for the hexagonal monocrystals, with the incident wave directed along the $z$-axis. The shorter path length through the thin plate results in a lower maximum magnitude of the internal field, while the longer path length through the prism results in a larger field with prominent focussing behaviour.

For the orientation shown in Fig. 12, the average and maximum magnitudes for the aggregate of 5 plates are quantified in Table 1. Since remote sensors probe an ensemble of particle orientations, and details of the far-field patterns are sensitive to orientation, we consider orientationally averaged far-field scattering quantities in the following sections.

\subsubsection{Far-field scattering}
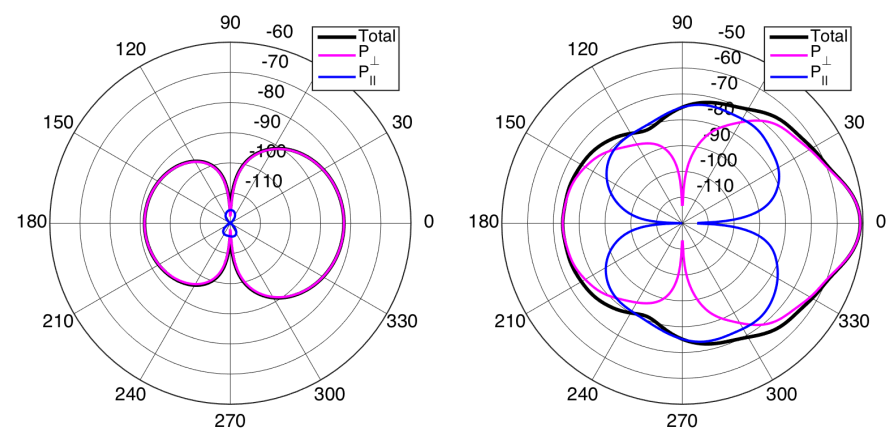

(a) $x=2$

(b) $x=10$

Figure 13: Scattering in different directions by chain-like aggregate of 5 plates for $(a) x=2$ and $(b) x=10$. In $(a)$, the scattering due to $P_{\perp}$ (magenta line) almost entirely overlaps the total scattering (black), and the amount of scattering due to $P_{\|}$(blue) is very small. For both size parameters, the results are averaged over 36 orientations in the $x-y$ plane.

The far-field scattering results for the aggregate of 5 plates is shown in Fig. 13. As in previous cases, the incident plane wave propagates in the $y$ direction, and is polarised perpendicular to the incident wave, along the $x$-axis. However, in this case we have considered an orientationally averaged example, for different orientations in the $x-y$ plane. The particle is rotated at intervals of $10^{\circ}$ about the $z$-axis, resulting in scattering calculations being obtained for 36 different orientations. The results averaged over the 36 orientations are shown in Fig. 13. As before, the cross sections have been computed using all components of the internal field, and also using only the components perpendicular and parallel to the incident wave, $P_{\perp}$ and $P_{\|}$.

For $x=2, P_{\perp}$ is responsible for the majority of the total scattering. The transverse component also has a larger contribution towards the total scattering value for $x=10$. Similar to the re- sults seen for the single plate with a fixed orientation in Fig. 4, $P_{\perp}$ contributes mainly to forward and backward scattering, and $P_{\|}$contributes more to sidescatter. This pattern persists through orientation averaging, and is not limited to a single particle orientation.

\subsection{Spheroids of equal $D_{\max }$ and aspect ratio to plate-like ag- gregates, but with effective permittivity determined by vol- ume fraction}

Comparisons have been done with the internal field of spheroids of equal $D_{\max }$ and aspect ratio to the aggregates of plates. The permittivity of the spheroids have been reduced according to the Maxwell-Garnett formula in Ref. [33]. This mixing ratio determines the volume fraction of ice that such a spheroid has, and subsequently calculates the corresponding effective permittivity. Such soft sphere and spheroid approximations have been used extensively in previous literature, so we are interested in assessing the performance of this method.

For brevity, the internal field plots have not been included here; instead we summarise the results briefly. In all cases examined it is found that the Maxwell-Garnett approximation overestimates the average internal field value. This overestimation ranges between $5 \%$ and $11 \%$. For the spheroidal approximation of 2 hexagonal plates of $x=2$, the maximum field value is also overestimated. However, as the particle size or complexity is increased, the Maxwell-Garnett approximation results in underestimations of the maximum value. This is caused by the incapability of the spheroid to replicate complex interactions within realistic particles.

\subsection{Irregular aggregates of 10 "fernlike dendrite" monomers}

Realistic snowflakes modelled by Tyynelä et al. [30] have been used to examine the internal field of more complex geometries. These particles are composed of 10 "fernlike dendrite" monomers. Ten different arrangements of these particles were available to us, with values of $D_{\max }$ ranging from $6-9 \mathrm{~mm}$. We plot the field of one arrangement in Fig. 14, and include results for the average and peak fields of two different arrangements in Table 1.

\subsubsection{Internal field}

In Fig. 14, the internal field of one aggregate is plotted for $x=2$ and $x=10$. Note that the range of the colour scale has been reduced for these particles to observe more detail in the internal field. For both values of $x$, the average field value is very similar (approximately $0.65-0.7$, see Table 1 ), and the magnitudes are lower than in all other particles considered. The maximum field value is also considerably smaller than in the different monocrystals of equal $x$. The same thing was found when these calculations were repeated for various different arrangements of 10 monomers. It can also be seen in Table 1 that calculations for $x=0.01$ show almost identical average and maximum values to $x=2$.

The values obtained for these particles are more similar to the value of $|\mathbf{E}| \approx 0.58$ you would see if only the applied field were present. This shows that for these fluffy aggregates, RGA 


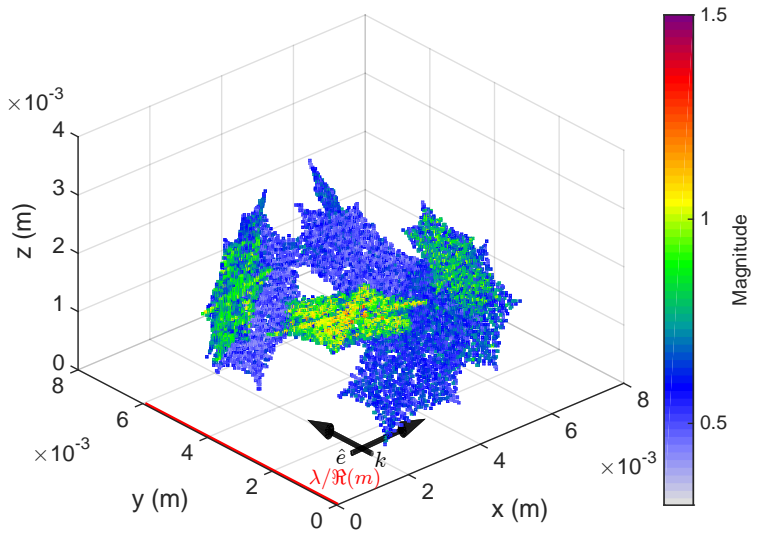

(a)

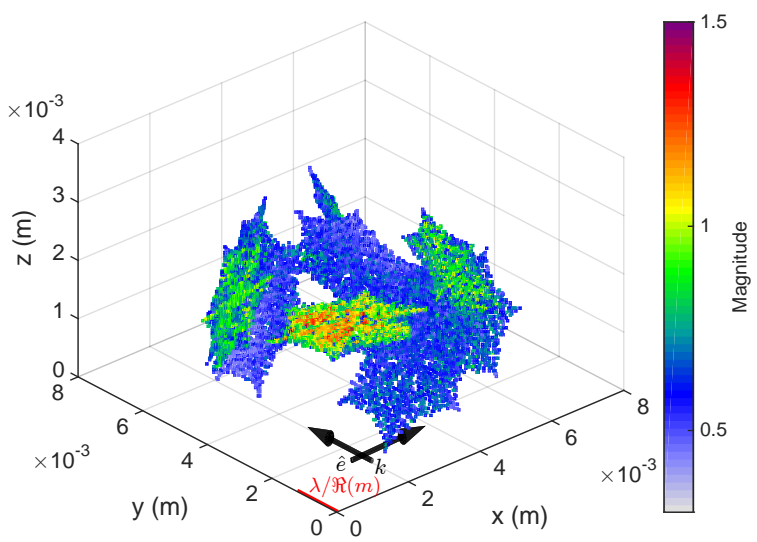

(b)

Figure 14: Magnitude of the internal field of an irregular aggregate of 10 "fernlike dendrite" monomers for $(a) x=2$ and $(b) x=10$. The dipole spacing is $47 \mu \mathrm{m}$, giving approximately $n_{\lambda}=125$ for $x=2$, and $n_{\lambda}=25$ for $x=10$. Note that the range of values in the colorbar is reduced, compared to previous cases.

is a more realistic approximation than for monocrystals. However, the true field is still systematically $15-20 \%$ higher than that assumed by RGA, while peak values are at least a factor 2 larger. These differences in the internal field strength lead to underestimates of scattering cross-sections by RGA, as observed by Tyynelä et al. [12].

No focussing behaviour is obvious for $x=2$ or $x=10$ in the complex aggregate. In fact, the individual crystals within each aggregate seem to be independent of each other, acting as if they are isolated. To test this, we calculated the field of the individual crystals detached from the rest of the aggregate. If we isolate crystals, we find the same field to within $5 \%$ of that calculated in the presence of the surrounding crystals. There is very little coupling between different monomers in the particle - only intramonomer. This may suggest a simplified method of computing scattering from large complex aggregates by considering interactions only within individual monomers. This implies that the "modified RGA" method developed by Lu et al. [52] is a reasonable approximation. In that method the range of interactions between dipoles is limited to some multiple of the minimum dimension of the particle. The method could provide good results for the fluffy aggregates considered here, provided that the range of interactions used is close to the scale of a monomer. We are developing this idea and testing it for a range of aggregates of different densities in a separate publication. This will help to clarify the range of applicability of such a method, and uncover whether it is limited to sparse aggregates, or if good results can also be obtained for rimed particles.

Our results show that if the scattering by the monomer crystals can be computed individually, the net scattering by the aggregate can quickly be estimated using RGA, since coupling between the monomers is small. For monomers small compared to the wavelength, the monomer scattering could be calculated rapidly using the results in Ref. [53]. For larger monomers, DDA calculations could be used (but on a much smaller scale than required to compute the scattering by the whole cluster). Our results support the assumptions made by Hogan et al. [54] who postulated that if an isolated monomer crystal scatters according to Gans theory with a particular dielectric factor (related to the internal field of the monomer), then a larger aggregate composed of several monomers could be described using RGA with that same value of dielectric factor - i.e. neglecting inter-monomer coupling.

\subsubsection{Far-field scattering}

For the complex aggregates, we have also considered a scenario averaged over 36 orientations, using the same methodology described in section 7.2.2 for the aggregate of 5 plates. The polar scattering plots in Fig. 15 show the orientationally averaged differential scattering cross sections. Panels (a) and (b) show the total amount of scattering, along with the contributions from the perpendicular and parallel components of the field, for $x=2$ and 10. The results exhibit similar behaviour to the aggregate of 5 plates in Fig. 13. As before, a significant amount of scattering in the forward and backward directions is due to the component of the field perpendicular to the incident wave, with the parallel component contributing mainly to sidescatter. For the larger size parameter of $x=10$, a comparison with the results for the aggregate of 5 plates in Fig. 13 shows that the influence of $P_{\|}$on the total amount of scattering is getting weaker with particle complexity. In contrast to the aggregate of plates, there are now very few angles where $P_{\|}$ dominates the total. The cross sections for individual crystals isolated from the aggregate were also examined, showing the same results. Therefore we suggest that the decrease in contribution from the component in the direction of propagation occurs as a result of the reduction in homogeneity of the particle composition, i.e. the presence of regions of air between solid ice branches.

It is worth noting that in contrast to the example of a symmetric plate, the $z$ component of the field for these fluffy aggregates is comparable to the $y$ component. Although the $z$ component is larger, it is not significant for the scattering quantity shown 
here as in Eq. (8) we chose the detector to be polarised in the $x$ $y$ plane. However, we did also consider single orientation cases with a $z$ polarised incident wave travelling in the $x$ and $y$ directions, with the detector polarised in the $x-z$ and $y-z$ directions, respectively. The corresponding results for $x=10$ are shown Fig. S16 of the supplementary material. The same conclusion applies to these cases - the component parallel to the propagation direction is small and contributes to sidescatter, while the transverse component is responsible for the majority of the scattering in the forward and backward directions. Orientational averaging was not done for these cases.

It is interesting to explore whether the diminishing contribution from the component parallel to propagation is a result of the inability of such low density structures to support a standing wave like that observed in the plot of $\mathfrak{R}\left(E_{y}\right)$ for the hexagonal plate in Fig. 3b. To test this, we plotted the parallel component of the field for some of the aggregate setups considered, i.e. for different incident directions and polarisations (not shown for brevity). It is found that there is no clear standing wave structure throughout the aggregate in any of the cases, suggesting that the standing wave may indeed play a key role in sidescatter. However, further work would be required to confirm whether the decreased influence of the field component parallel to the propagation direction on the total amount of scattering within a complex aggregate is in fact caused by the inability to maintain a standing wave on the perimeter.

\subsection{Sphere of equal $D_{\max }$ to aggregate, but with effective per- mittivity determined by volume fraction}

In a similar manner to section 7.3, the Maxwell-Garnett formula is used to calculate the internal field of a sphere of equal $D_{\max }$ to the aggregate of fernlike dendrites. The plots of the field have been omitted in the interest of brevity. It is found that approximating the aggregate by a soft sphere results in a more uniform internal field. The symmetry of the particle leads to very slight focussing behaviour towards the forward region, but the magnitude of the field is close to 1 everywhere, meaning the average field value is larger than in the aggregate. The maximum magnitude value in the sphere is lower than in the aggregate, and the minimum value is higher.

In panels (c) and (d) of Fig. 15, the far field scattering by the complex aggregate is compared to the result calculated using the equivalent sphere, and also using RGA. For $x=2$, the averaged differential cross section, $\sigma$, is accurately approximated by the equivalent sphere in the forward direction. However, the approximation overestimates sidescatter and underestimates backscatter for this size parameter. For $x=10$, the DDA result is underestimated at almost all scattering angles by the equivalent sphere, particularly in the backscatter direction. Such underestimates in scattering properties as a result of soft sphere approximations are consistent with previous literature, e.g. [55, 56], with Ref. [56] showing that horizontally aligned soft spheroids provide better results for radar scattering by ice clouds. However, Tyynelä et al. [30] found that soft spheroids also underestimate the backscatter cross section of realistic aggregates.
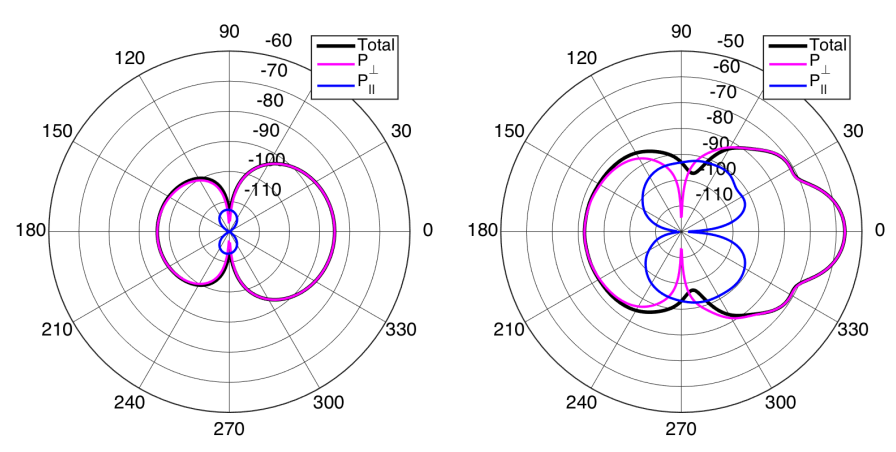

(a) $x=2$

(b) $x=10$
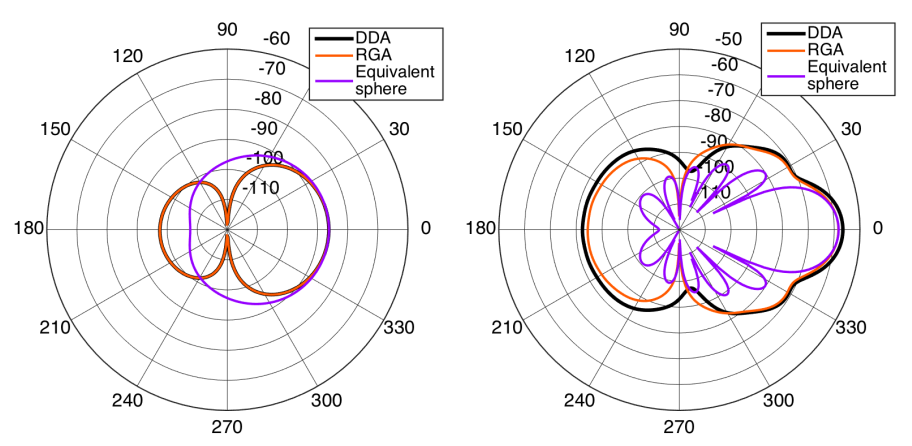

(c) $x=2$

(d) $x=10$

Figure 15: Orientationally averaged scattering cross section at different scattering angles by a complex aggregate for $(a, c) x=2$ and $(b, d) x=10$. The black lines in all plots show the total amount of scattering. The magenta lines in the top row show scattering due to $P_{\perp}$, and the blue lines show scattering due to $P_{\|}$. Panels $(c)$ and $(d)$ show comparisons of the total scattering with results obtained using RGA (orange), and an equivalent sphere approximation (purple) using the Maxwell-Garnett mixing ratio. The DDA and RGA results are almost identical for $x=2$, with the orange line covering the black line in panel (c).

The inaccuracies that result from soft sphere and spheroid approximations are due to a combination of 2 factors. As we just discussed, the first reason is that the internal fields are not represented correctly, with the Maxwell-Garnett approximation leading to a less structured field. The second reason is that the spatial structure of ice is incorrect when approximating a realistic particle by a spherical or spheroidal equivalent. It has been suggested that for sparse structures such as the fluffy aggregates we are interested in, using RGA could result in more accurate scattering calculations than soft sphere or spheroid approximations, as in Ref. [12]. Since RGA doesn't include internal field interactions, these improvements to scattering by aggregates have been attributed to the fact that the spatial structure of the particle is modelled in RGA calculations. Fig. 15 (c) and (d) show $\sigma$ for the aggregates of "fernlike dendrites" using RGA. It is clear that significant improvements are indeed seen for the irregular geometries used in this study by using RGA rather than soft sphere and spheroid approximations. Thus, a significant amount of the error caused by those approximations is due to 
the incorrect representation of the spatial structure of the particle. Internal coupling plays a smaller role, but nonetheless a notable one. Similar to the findings in Ref. [12], large errors between RGA and DDA are found in some directions. Underestimations of the backscatter cross sections become more prominent with size parameter, reaching $-38 \%$ for $x=10$, with errors increasing further towards sidescattering angles. Comparisons of the internal fields of these aggregates show that the average field strength is higher using DDA than it is using RGA. As the internal field is the same everywhere using RGA, the structure is also noticeably different. Hence it is worthwhile exploring whether improvements can be made to RGA in order to represent some internal structure and improve scattering calculations.

\section{Conclusions}

In this paper we use the DDA numerical method to investigate the internal field and scattering properties of ice particles, presenting results for two different size parameters of $x=2$ and $x=10$. We also reference values obtained for some of the particles with a smaller size parameter of $x=0.01$, where the particle is in the Rayleigh regime. In section 5.2, we point out that convergence is slow with increased grid resolution. It has been suggested that such convergence issues could be improved by weighting the discretisation of dipoles on the edge of the particle, such as the method used in Ref. [57], and we plan to investigate this in future work.

Exploring the magnitude of the internal electric field for different monocrystals, it is found that the field varies greatly with size parameter. For $x=0.01$, the field is almost uniform, with a factor of 1.15 between the average and maximum field values of the hexagonal plate. The magnitude of the field is small, with an average of approximately 1.4 times the value obtained as a result of the applied wave only. For $x=2$ the magnitude is also small with a relatively uniform field, ranging from an average of approximately 1.02 for the hexagonal prism, to approximately 1.13 for the cylindrical disk. Increasing $x$ results in a more complex internal field with larger maximum values. Strong focussing is observed at the forward side of the monocrystals for $x=10$. The focussing behaviour is persistent for different orientations in the $x-y$ plane, and occurs independently of whether the incident wave hits a flat prism facet or a sharp edge. Strong focussing remains when the incident wave is directed at small angles in the $y-z$ plane, but the behaviour starts to diminish for larger angles. Such angles correspond to cases where the incident wave is approximately travelling in the direction of a basal face, rather than hitting a prism face or edge. The diminished focussing is a result of the small thickness of the plate in the direction of travel of the incident wave, as strong focussing is found when the same angles are used along with the thicker hexagonal prism of aspect ratio 1. For the single hexagonal plate presented in this manuscript, the internal field structure is a combination of 2 distinct waves. The perpendicular component of the field, $P_{x}$, displays a wave extending through the centre of the particle, and the parallel component, $P_{y}$, displays a standing wave around the perimeter.
Mitchell and co-workers developed the Modified Anomalous Diffraction Approximation ("MADA"), for the efficient prediction of the extinction of radiation by water droplets [58] and ice crystals [59] at size parameters $x \approx 10-1000$. In addition to its speed, an attractive feature of the MADA is that physical wave scattering phenomena are explicitly represented as separate terms, and this can provide insight into their roles in the scattering process. Our results provide new data on these scattering phenomena for hexagonal ice crystals (specifically the existence and characteristics of internally-reflected surface waves and waves extending through the particle), and thus may be informative for further development and theoretical underpinning of the MADA approach.

A simple aggregate of 2 hexagonal plates sees a similar uniformity of the internal field for $x=0.01$ and $x=2$. However, a dramatic decrease in focussing behaviour and symmetry is observed for $x=10$, along with an overall smoothing of the field. This becomes even more obvious for a chain-like aggregate of 5 plates. It is noted that this behaviour is partly controlled by the alignment of the plates, and the focussing patterns vary depending on the arrangement of the individual monocrystals (see Ref. [41], in preparation).

As the complexity of the particle is increased further, the internal field continues to lose more structure, and no focussing behaviour is obvious for the fluffy aggregate of 10 dendrites. The maximum value of electric field decreases significantly for aggregated dendrites, with the single hexagonal plate displaying a maximum magnitude approximately 3 times larger than the irregular aggregates for $x=10$. Different values of $x$ give very similar internal fields for the complex aggregate, with almost identical average and maximum values for $x=0.01$ and $x=2$, and slightly larger values for $x=10$. An interesting observation is that the individual dendrites comprising the aggregate act somewhat independently of each other, as seen by calculating the field of the monocrystals in isolation from the rest of the particle. This could have important consequences for scattering calculations, suggesting that calculations for large aggregates could be done without the need for huge computer resources. Individual crystals could be solved independently and then combined to obtain approximations for complex particles.

To explore the performance of currently used approximations, the Maxwell-Garnett formula has been employed to calculate the internal field of spherical or spheroidal particles of equivalent size to the different aggregates in this study. It is found that the reduced permittivity used in this method leads to the loss of internal field structure. The effective medium approximation causes the average field to be overestimated for all particles, and the maximum value to be underestimated in most cases.

The effect of the internal electric field on far-field scattering is also examined by calculating the differential scattering cross section for a number of particles in this study. For $x=0.01$, the particles scatter equally in the forward and backward directions, and can be represented fully using only the component of the field which is perpendicular to the direction of propaga- 
tion. As size parameter is increased, the amount of scattering observed becomes very dependent on scattering angle. For both single orientation and orientationally averaged cases, it is found that the component perpendicular to the direction of propagation contributes mainly to scattering in the forward and backward directions. For size parameters of $x=2$ and $x=10$, a field component in the direction of propagation emerges, and this parallel component contributes to sidescatter. It is evident that for $x=10$, the influence of the latter component is getting weaker as particle complexity is increased. The polar plot for the irregular aggregate displays very few angles where $P_{\|}$ dominates the total scattering. We saw for the hexagonal plate that it is the standing wave around the perimeter that leads to sidescatter. For irregular aggregates of "fernlike" dendrites, it appears that their fluffy structure is incapable of supporting such a standing wave. This may be why we see a decrease in the contribution from $P_{\|}$to the total scattering.

Approximations of dendritic aggregates using MaxwellGarnett soft spheres is explored. As discussed, this method leads to an internal field that is more uniform than the true field. In the far-field, underestimations of scattering properties are found. The far field results are also calculated using RGA, showing more accurate results than the soft sphere, but with errors persisting at some angles for $x=10$, predominantly in the backward hemisphere.

Of course this is an idealised scenario as the majority of the work presented here considers particles of fixed orientation. On one hand it is a scenario that makes sense physically, as planar crystals tend to orient themselves horizontally [60]. Hence, the fixed orientation examples for plate-like particles are similar to probing with radar at low elevation. However, we acknowledge that plates may oscillate and rotate around the axis perpendicular to their face, depending on the Reynolds number [61]. Therefore, the geometries presented in this manuscript and in the supplementary material are unlikely to be representative of all orientations. Although we considered orientationally averaged results for the complex aggregate, it would be interesting to develop this work by looking at orientationally averaged examples for all particles. Another interesting extension to this work would be to investigate the evolution of the internal field as the size parameter is increased to much larger values, which may possible for infinitely long, thin crystals, as described in Refs. [62, 63].

\section{Acknowledgments}

This work was supported financially by a studentship from the Engineering and Physical Sciences Research Council (EPSRC), and the UK Met Office. We thank Jani Tyynelä for valuable discussions on internal field structure, and for providing the dendritic aggregate models. We also thank Sam Groth for help with the BEM++ numerical method, and Anthony Baran and Simon Chandler-Wilde for useful discussions. We extend our gratitude to the three reviewers for very useful suggestions.

The data that support the findings of this study are available from the corresponding author, KMC, upon request.

\section{References}

[1] Mülmenstädt, J., O. Sourdeval, J. Delanoë, and J. Quaas, 2015: Frequency of occurrence of rain from liquid-, mixed-, and ice-phase clouds derived from A-Train satellite retrievals. Geophys. Res. Lett., 42, 65026509. https://doi.org/10.1002/2015GL064604

[2] Sourdeval, O., Labonnote L. C., Baran A. J., Mülmenstädt, J., and Brogniez, G., 2016: A methodology for simultaneous retrieval of ice and liquid water cloud properties. Part 2: Near-global retrievals and evaluation against A-Train products. Q. J. R. Meteorol. Soc., 142, 3063-3081. https://doi.org/10.1002/qj.2889

[3] Eliasson, S., S. A. Buehler, M. Milz, P. Eriksson, and V. O. John, 2011: Assessing observed and modelled spatial distributions of ice water path using satellite data. Atmos. Chem. Phys., 11, 375-391. https://doi.org/10.5194/acp-11-375-2011

[4] Stephens, G. L., and Coauthors, 2008: CloudSat mission: Performance and early science after the first year of operation. J. Geophys. Res., 113. https://doi.org/10.1029/2008JD009982

[5] Wood, N. B., 2011: Estimation of snow microphysical properties with application to millimeter-wavelength radar retrievals for snowfall rate. $\mathrm{PhD}$ Thesis, Colorado State University, 231 pp.

[6] Evans, K. F., J. R. Wang, P. E. Racette, G. Heymsfield, and L. Li, 2005: Ice cloud retrievals and analysis with the compact scanning submillimeter imaging radiometer and the cloud radar system during CRYSTAL FACE. J. Appl. Meteor., 44, 839-859. https://doi.org/10.1175/JAM2250.1

[7] Brath, M., S. Fox, P. Eriksson, R. C. Harlow, M. Burgdorf, and S. A. Buehler, 2018: Retrieval of an ice water path over the ocean from ISMAR and MARSS millimeter and submillimeter brightness temperatures. Atmos. Meas. Tech., 11, 611-632. https://doi.org/10.5194/amt-11-611-2018

[8] Kangas, V., and Coauthors, 2014: Ice cloud imager instrument for MetOp second generation. 13th Spec. Meeting Microw. Radiometry Remote Sens. Environ., pp. 228-231. http://dx.doi.org/10.1109/MicroRad.2014.6878946

[9] Eriksson, P., S. A. Buehler, C. P. Davis, C. Emde, and O. Lemke, 2011: ARTS, the atmospheric radiative transfer simulator, version 2. J. Quant. Spectrosc. Radiat. Transfer, 112(10), 1551-1558. https://doi.org/10.1016/j.jqsrt.2011.03.001

[10] Eriksson, P., Ekelund, R., Mendrok, J., Brath, M., Lemke, O., and Buehler, S. A., 2018: A general database of hydrometeor single scattering properties at microwave and sub-millimetre wavelengths. Earth Syst. Sci. Data, 10, 1301-1326. https://doi.org/10.5194/essd-10-1301-2018

[11] Westbrook, C. D., R. C. Ball, and P. R. Field, 2006: Radar scattering by aggregate snowflakes. Q. J. R. Meteorol. Soc., 132(616), 897-914. https://doi.org/10.1256/qj.05.82

[12] Tyynelä, J., J. Leinonen, C. D. Westbrook, D. Moisseev, and T. Nousiainen, 2013: Applicability of the Rayleigh-Gans approximation for scattering by snowflakes at microwave frequencies in vertical incidence. J. Geophys. Res. Atmos., 118, 1826-1839. https://doi.org/10.1002/jgrd.50167

[13] Leinonen, J., S. Kneifel, and R. J. Hogan, 2017: Evaluation of the Rayleigh-Gans approximation for microwave scattering by rimed snowflakes. Q. J. R. Meteorol. Soc., 144(S1), 77-88. https://doi.org/10.1002/qj.3093

[14] Chen, Z., A. Taflove, and V. Backman, 2004: Photonic nanojet enhancement of backscattering of light by nanoparticles: a potential novel visible-light ultramicroscopy technique. Opt. Express, 12(7), 1214-1220. https://doi.org/10.1364/OPEX.12.001214

[15] Mendes, M. J., I. Tobas, A. Mart, and A. Luque, 2011: Light concentration in the near-field of dielectric spheroidal particles with mesoscopic sizes. Opt. Express, 19, 16207-16222. https://doi.org/10.1364/OE.19.016207

[16] Owen, J. F., R. K. Chang, and P. W. Barber, 1981: Internal electric field distributions of a dielectric cylinder at resonance wavelengths. Opt. Lett., 6, 540-542. https://doi.org/10.1364/OL.6.000540

[17] Tyynelä, J., 2011: Polarization studies in electromagnetic scattering by small Solar-system particles. PhD Thesis, University of Helsinki, 57 pp.

[18] Tyynelä, J., E. Zubko, K. Muinonen, and G. Videen, 2010: Interpretation of single-particle negative polarization at intermediate scattering angles. Appl. Opt., 49, 5284-5296. https://doi.org/10.1364/AO.49.005284

[19] Barton, J. P., 2002: Electromagnetic field calculations for an irregularly shaped, near-spheroidal particle with arbitrary illumination. JOSA, 19, 2429-2435. https://doi.org/10.1364/JOSAA.19.002429 
[20] Lu, Y., E. E. Clothiaux, K. Aydin, G. Botta, and J. Verlinde, 2013: Modeling variability in dendritic ice crystal backscattering cross sections at millimeter wavelengths using a modified RayleighGans theory. J. Quant. Spectros. Radiat. Transfer, 131, 95-104. https://doi.org/10.1016/j.jqsrt.2013.05.008

[21] Westbrook, C. D., A. J. Illingworth, E. J. O'Connor and R. J. Hogan, 2010: Doppler lidar measurements of oriented planar ice crystals falling from supercooled and glaciated layer clouds. Q. J. R. Meteorol. Soc., 136, 260-276. https://doi.org/10.1002/qj.528

[22] Platt, C. M., N. L. Abshire, and G. T. McNice, 1978: Some Microphysical Properties of an Ice Cloud from Lidar Observation of Horizontally Oriented Crystals. J. Appl. Meteor., 17, 1220-1224. https://doi.org/10.1175/1520-0450(1978)017<1220:SMPOAI >2.0.CO;2

[23] Heymsfield, A. J., and C. M. R. Platt, 1984: A Parameterization of the Particle Size Spectrum of Ice Clouds in Terms of the Ambient Temperature and the Ice Water Content. J. Atmos. Sci., 41, 846-855. https://doi.org/10.1175/1520-0469(1984)041<0846:APOTPS > 2.0.CO;2

[24] Um J., G. M. McFarquhar, Y. P. Hong, S.-S. Lee, C. H. Jung, R. P. Lawson, Q. Mo, 2015: Dimensions and aspect ratios of natural ice crystals. Atm. Chem. Phys., 15(7), 3933-3956. https://doi.org/10.5194/acp-15-3933-2015

[25] Bailey, M. P. and J. Hallett, 2009: A Comprehensive Habit Diagram for Atmospheric Ice Crystals: Confirmation from the Laboratory, AIRS II, and Other Field Studies. J. Atmos. Sci., 66, 2888-2899. https://doi.org/10.1175/2009JAS2883.1

[26] Stith, J. L., J. Dye, A. Bansemer, A. J. Heymsfield, C. A. Grainger, W. A. Petersen, and R. Ciffelli, 2002: Microphysical observations of tropical clouds. J. Appl. Meteorol., 41, 97-117. https://doi.org/10.1175/1520-0450(2002)041<0097:MOOTC>2.0.CO;2

[27] Stith, J. L., J. A. Haggerty, A. Heymsfield, and C. A. Grainger, 2004: Microphysical Characteristics of Tropical Updrafts in Clean Conditions. J. Appl. Meteor., 43, 779-794. https://doi.org/10.1175/2104.1

[28] Connolly, P. J., C. P. R. Saunders, M. W. Gallagher, K. N. Bower, M. J. Flynn, T. W. Choularton, J. Whiteway, and P. Lawson, 2005 Aircraft observations of the influence of electric fields on the aggregation of ice crystals. Q. J. R. Meteorol. Soc., 131, 1695-1712. https://doi.org/10.1256/qj.03.217

[29] Hobbs, P. V., S. Chang, and J. D. Locatelli,1974: The Dimensions and Aggregation of Ice Crystals in Natural Clouds. J. Geophys. Res., 79, 21992206. https://doi.org/10.1029/JC079i015p02199

[30] Tyynelä, J., J. Leinonen, D. Moisseev, and T. Nousiainen, 2011: Radar backscattering from snowflakes: Comparison of fractal, aggregate, and soft spheroid models. J. Atmos. Oceanic Technol., 28(11), 1365-1372. https://doi.org/10.1175/JTECH-D-11-00004.1

[31] Eriksson, P., M. Jamali, J. Mendrok, and S. A. Buehler, 2015: On the microwave optical properties of randomly oriented ice hydrometeors. Atmos. Meas. Tech., 8, 1913-1933. https://doi.org/10.5194/amt-8-1913-2015

[32] Mätzler, C., 2006: Microwave dielectric properties of ice. Thermal microwave radiation - Applications for remote sensing, C. Mätzler, Inst. Eng. Technol., Stevenage, UK, 455-462.

[33] Maxwell-Garnett, J. C., 1904: Colours in metal glasses and in metallic films. Philos. Trans. Roy. Soc. London, 203, 385-420. http://dx.doi.org/10.1098/rsta.1904.0024

[34] Purcell, E. M., and C. R. Pennypacker, 1973: Scattering and absorption of light by non-spherical dielectric grains. Astrophys. J., 186, 705-714. http://dx.doi.org/10.1086/152538

[35] Draine, B. T., and P. J. Flatau, 1994: Discrete-dipole approximation for scattering calculations. J. Opt. Soc. Amer., 11A, 1491-1499. http://dx.doi.org/10.1364/JOSAA.11.001491

[36] Draine, B. T., and J. Goodman, 1993: Beyond Clausius-Mossotti - Wave propagation on a polarizable point lattice and the discrete dipole approximation. Astrophys. J., 405, 685-697. http://dx.doi.org/10.1086/172396

[37] Yurkin, M. A., and A. G. Hoekstra, 2007: The discrete dipole approximation: An overview and recent developments. J. Quant. Spectros. Radiat. Transfer, 106(1-3), 558-589. http://dx.doi.org/10.1016/j.jqsrt.2007.01.034

[38] Petty, G.W., and W. Huang, 2010: Microwave backscatter and extinction by soft ice spheres and complex snow aggregates. J. Atmos. Sci., 67, 769787. https://doi.org/10.1175/2009JAS3146.1

[39] Zubko, E., D. Petrov, Y. Grynko, Y. Shkuratov, H. Okamoto, K. Muinonen, T. Nousiainen, H. Kimura, T. Yamamoto, and G. Videen,
2010: Validity criteria of the discrete dipole approximation. Appl. Opt., 49, 1267-1279. https://doi.org/10.1364/AO.49.001267

[40] Draine, B.T., and P. J. Flatau, 2010: "User Guide for the Discrete Dipole Approximation Code DDSCAT 7.1". http://arxiv.org/abs/1002.1505

[41] McCusker, K., 2019 (in preparation): Fast, approximate methods for electromagnetic wave scattering by complex ice crystals and snowflakes. $\mathrm{PhD}$ Thesis, University of Reading.

[42] Yurkin, M. A., V. P. Maltsev, and A. G. Hoekstra, 2007: The discrete dipole approximation for simulation of light scattering by particles much larger than the wavelength. J. Quant. Spectrosc. Radiat., 106, 546-557. doi: 10.1016/j.jqsrt.2007.01.033

[43] Groth, S.P., A. J. Baran, T. Betcke, S. Havemann, W. Śmigaj, 2015: The boundary element method for light scattering by ice crystals and its implementation in BEM++. J. Quant. Spectrosc. Radiat., 167, 40-52. https://doi.org/10.1016/j.jqsrt.2015.08.001

[44] Śmigaj, W., T. Betcke, S. Arridge, J. Phillips, and M. Schweiger, 2015: Solving boundary integral problems with BEM++. ACM Trans. Math. Softw., 41(2) Article no. 6. http://dx.doi.org/10.1145/2590830

[45] Yurkin, M. A., V. P. Maltsev, and A. G. Hoekstra, 2006: Convergence of the discrete dipole approximation. I. Theoretical analysis. J. Opt. Soc. Am. A, 23, 2578-2591. http://dx.doi.org/10.1364/JOSAA.23.002578

[46] Baran, A. J., E. Hesse, O. Sourdeval, 2017: The applicability of physical optics in the millimetre and sub-millimetre spectral region. Part I: The ray tracing with diffraction on facets method. J. Quant. Spectros. Radiat. Transfer, 190, 13-25. http://dx.doi.org/10.1016/j.jqsrt.2016.12.030

[47] Lu, Y., 2013: Development and analysis of an ice crystal scattering database for remote sensing applications and cloud model evaluation. $\mathrm{PhD}$ Thesis, The Pennsylvania State University, $125 \mathrm{pp}$.

[48] Tyynelä, J., E. Zubko, G. Videen, and K. Muinonen, 2007: Interrelating angular scattering characteristics to internal electric fields for wavelengthscale spherical particles. J. Quant. Spectrosc. Radiat., 106, 520-534. https://doi.org/10.1016/j.jqsrt.2007.01.032

[49] Muinonen, K., Tyynelä, J., Zubko, E., Lindqvist, H., Penttilä, A., Videen, G., 2011: Polarization of light backscattered by small particles. J. Quant. Spectrosc. Radiat., 112, 2193-2212. https://doi.org/10.1016/j.jqsrt.2011.06.009

[50] Matrosov, S. Y., A. J. Heymsfield, and Z. Wang, 2005: Dual-frequency radar ratio of nonspherical atmospheric hydrometeors. Geophys. Res. Lett., 32, L13816. https://doi.org/10.1029/2005GL023210

[51] McCusker, K., 2015: How fast do ice crystals grow? MSc. Dissertation, Department of Meteorology, University of Reading, 49 pp. https://icecrystalsblog.files.wordpress.com/2018/06/dissertation-finalcopy.pdf

[52] Lu, Y., E. E. Clothiaux, K. Aydin, and J. Verlinde, 2014: Estimating ice particle scattering properties using a modified RayleighGans approximation. J. Geophys. Res. Atmos., 119, 10,471-10,484. doi:10.1002/2014JD021850

[53] Westbrook, C. D., 2014: Rayleigh scattering by hexagonal ice crystals and the interpretation of dual-polarisation radar measurements. $Q$. J. R. Meteorol. Soc., 140, 2090-2096. https://doi.org/10.1002/qj.2262

[54] Hogan, R. J., R. Honeyager, J. Tyynelä, and S. Kneifel, 2017: Calculating the millimetre-wave scattering phase function of snowflakes using the self-similar Rayleigh-Gans Approximation. Q. J. R. Meteorol. Soc., 143, 834-844. https://doi.org/10.1002/qj.2968

[55] Ori, D., T. Maestri, R. Rizzi, D. Cimini, M. Montopoli, and F. S. Marzano, 2014: Scattering properties of modeled complex snowflakes and mixedphase particles at microwave and millimeter frequencies. J. Geophys. Res. Atmos., 119, 9931-9947. https://doi.org/10.1002/2014JD021616

[56] Hogan, R. J., L. Tian, P. R. A. Brown, C. D. Westbrook, A. J. Heymsfield, and J. D. Eastment, 2012: Radar scattering from ice aggregates using the horizontally aligned oblate spheroid approximation. J. Appl. Meteorol. Climatol., 51(3), 655-671. https://doi.org/10.1175/JAMC-D-11-074.1

[57] Piller, N. B., 1997: Influence of the edge meshes on the accuracy of the coupled-dipole approximation. Opt. Lett., 22, 1674-1676. http://dx.doi.org/10.1364/OL.22.001674

[58] Mitchell, D.L., 2000: Parameterization of the Mie Extinction and Absorption Coefficients for Water Clouds. J. Atmos. Sci., 57, 1311-1326.

[59] Mitchell, D.L., Y. Liu, and A. Macke, 1996: Modeling Cirrus Clouds. Part II: Treatment of Radiative Properties. J. Atmos. Sci., 53, 2967-2988.

[60] Noel, V., and K. Sassen, 2005: Study of Planar Ice Crystal Orientations in Ice Clouds from Scanning Polarization Lidar Observations. J. Appl. 
Meteorol., 44, 653-664. https://doi.org/10.1175/JAM2223.1

[61] Cheng, K., P. K. Wang, and T. Hashino, 2015: A Numerical Study on the Attitudes and Aerodynamics of Freely Falling Hexagonal Ice Plates. J. Atmos. Sci., 72, 3685-3698. https://doi.org/10.1175/JAS-D-15-0059.1

[62] Flatau, P. J., and B. T. Draine, 2014: Light scattering by hexagonal columns in the discrete dipole approximation. Opt. Express, 22(18), 21834-21846. https://doi.org/10.1364/OE.22.021834

[63] Flatau, P. J., and B. T. Draine, 2012: Fast near field calculations in the discrete dipole approximation for regular rectilinear grids. Opt. Express, 20(2), 1247-1252. https://doi.org/10.1364/OE.20.001247 